\title{
A PHOTOMETRIC STUDY OF FIVE OPEN CLUSTERS IN THE SDSS
}

\author{
Jinhyuk Ryu and Myung Gyoon Lee \\ Astronomy Program, Department of Physics and Astronomy, Seoul National University, Seoul 151-742, Korea \\ E-mail : ryujh@astro.snu.ac.krandmglee@astro.snu.ac.kr \\ (Received July 19, 2011; Revised September 6, 2011; Accepted September 17, 2011)
}

\begin{abstract}
We present a photometric study of five open clusters (Czernik 5, Alessi 53, Berkeley 49, Berkeley 84, and Pfleiderer 3 ) in the Sloan Digital Sky Survey. The position and size of these clusters are determined using the radial number density profiles of the stars, and the member stars of the clusters are selected using the proper motion data in the literature. We estimate the reddening, distance and age of the clusters based on the isochrone fitting in the color-magnitude diagram. The foreground reddenings for these clusters are estimated to be $E(B-V)=0.71-1.55 \mathrm{mag}$. The distances to these clusters are derived to be $2.0-4.4 \mathrm{kpc}$, and their distances from the Galactic center range from $7.57 \mathrm{kpc}$ to 12.35 kpc. Their ages are in the range from $250 \mathrm{Myr}$ to 1 Gyr. Berkeley 49 and Berkeley 84 are located in the Orion spur, Czernik 5 is in the Perseus arm, and Pfleiderer 3 and Alessi 53 are located beyond the Perseus arm.
\end{abstract}

Key words : Galaxy: stellar content - Hertzsprung-Russell and C-M diagrams - open clusters and associations: general - open clusters and associations: individual (Alessi 53, Berkeley 49, Berkeley 84, Czernik 5, Pfleiderer 3)

\section{INTRODUCTION}

Open clusters (OCs) are groups of tens to a few thousands of stars with sizes of several parsecs. Stars in an OC are born simultaneously in a giant molecular cloud, which is usually found on the Galactic disk. Therefore, these OCs are excellent tracers of the formation and evolution of the Galactic disk. As an example, Piatti (2010) found that the age distribution of the OCs in our Galaxy shows two prominent peaks at 10-15 Myrs and 1.5 Gyrs, providing an evidence of enhanced formation episodes of the OCs at these epochs. There are more than two thousand OCs and OC candidates listed in the catalog of optically visible open clusters and candidates by Dias, Alessi, Moitinho, and Lépine (2002) (hereafter DAML02*). However, according to DAML02, physical parameters such as the reddening, distance and age of the clusters are still unknown for about half of them. Therefore, it is necessary to determine the physical parameters of these OCs for enhancing our knowledge about the Galaxy structure.

To determine the physical parameters of OCs, we can use photometry for individual OCs that have been observed by ourselves (Lee 1997; Park \& Lee 1999; Ann et al. 2002). However, it is less efficient than using wide field survey data which contains an enormous amount of data covering a large area of the sky. One of the widely used surveys is the Two Micron All Sky Survey (2MASS; Skrutskie et al. 2006). There are many

Corresponding Author: M. G. Lee

* Last updated at November 24th, 2010. studies about OCs based on the 2MASS, including discoveries of new OC candidates (Kronberger et al. 2006; Froebrich et al. 2007; Koposov et al. 2008), and estimations of physical parameters of OCs (Tadross 2008; Tadross 2009; Camargo et al. 2009).

The Sloan Digital Sky Survey (SDSS; York et al. 2000) is another excellent resource for the OC study. The SDSS covers a quarter of the whole sky and contains a few hundred million stars. Nevertheless, there are only a few studies about OCs using the SDSS until now. For example, An et al. (2008) presented fiducial sequences of 17 Galactic globular clusters and 3 wellknown OCs (NGC 2420, NGC 6791 and M67), and An et al. (2009) made theoretical isochrones and compared them with data in An et al. (2008).

In this paper, using the SDSS, we present a photometric study of five OCs in our Galaxy that have been less studied: Czernik 5, Alessi 53, Berkeley 49, Berkeley 84 and Pfleiderer 3. We determine the physical parameters of these clusters, using the ugriz photometry we derive from the SDSS images. Three of them (Czernik 5, Berkeley 49 and Berkeley 84) were studied previously using 2MASS data (Tadross 2008; Tadross 2009; Camargo et al. 2009), but none of them were studied in the optical band. During this study, Subramaniam et al. (2010) presented BVI photometry of Berkeley 49. No study is available for Alessi 53 and Pfleiderer 3 as yet.

This paper is organized as follows. Section 2 describes the data, selection of target clusters and the procedure for deriving the photometry of the target 
clusters. Section 3 explains the methods used to determine the center and size of each cluster, and to derive physical parameters of the clusters. In Section 4, we present color-magnitude diagrams (CMD) of these clusters. Then we derive foreground reddening, distance and age of these clusters from the CMDs. We discuss the properties of the target clusters, and their locations in the Milky Way in Section 5. Finally primary results are summarized in Section 6.

\section{DATA AND DATA REDUCTION}

We used $u, g, r, i$, and $z$ images in the SDSS for this study. The SDSS obtains images and spectra of sources using a 2.5-m telescope at the Apache Point Observatory. It covers a quarter of the entire sky for survey, and the limiting magnitudes of photometry are $22.0,22.2,22.2,21.3$, and $20.5 \mathrm{mag}$ for $u, g, r, i$, and $z$ bands, respectively. These magnitudes correspond to $95 \%$ detection repeatability for point sources. The field of view for a chip is $13^{\prime} .51 \times 8^{\prime} .98$, and the pixel scale is $0^{\prime \prime} .396$. An image is taken by the drift scan with an integration time of 54 seconds.

We selected the target clusters from among 170 OCs in the SDSS Data Release 7 (DR7; Abazajian et al. 2009) with the following criteria: (1) location far from the edge of the survey area, (2) must be distinguished clearly from backgrounds, (3) must be poorly studied, (4) not be saturated, and (5) not having too large angular size (smaller than $\sim 10^{\prime}$ ). Finally, we chose 5 OCs: Czernik 5, Alessi 53, Berkeley 49, Berkeley 84 and Pfleiderer 3.

The SDSS provides point-spread-function (PSF) fitting magnitudes of point sources. However, standard SDSS photometric pipelines (Lupton et al. 2002) do not provide PSF magnitudes of some stars in the crowded regions such as the central region of a star cluster. Therefore, we decided to derive photometry of these clusters by ourselves rather than using the SDSS DR7 catalog.

We derived PSF magnitudes from the SDSS images using the $\operatorname{IRAF}^{\dagger} /$ DAOPHOT (Stetson 1987). We obtained FITS files of the chosen clusters from the SDSS Data Archive Server (DAS). These images were already bias subtracted, dark corrected and flat fielded by pipelines.

Fig. 1 shows gray-scale maps of $r$ band images of the clusters. Pfleiderer 3 was observed twice, and we combined the two images. For each field containing the clusters, we derived photometry independently. We used the gain and readout noise of the SDSS chips from Table 4 of An et al. (2008). After deriving the magnitudes in each band, we selected point sources that are detected at least in two bands.

†IRAF is distributed by NOAO, which are operated by the Association of Universities for Research in Astronomy, Inc. under contract with the National Science Foundation.
We derived zero-point differences using the sources that are $r \leq 17$ and common between ours and the SDSS DR7 point source catalogs. Zero-points, rms errors, and the number of stars used for the standard calibration are listed in Table 1. All rms errors of the standard calibration is less than 0.004. Table 2 lists the ugriz photometry of these five clusters.

\section{METHOD}

\subsection{Membership Determination}

We selected member stars of the clusters using proper motion data in the PPMXL (Roeser et al. 2010b). The PPMXL is an astrometric catalog prepared by combining USNO-B1.0 and the 2MASS astrometry. The catalog provides positions and absolute proper motions. It is complete down to about $V=20$ mag. The average and dispersion of the proper motions were estimated using the stars in the field of each cluster with the following conditions: $\mathrm{J}<14$, $R \leq 2 R_{\mathrm{cl}}$, where $R_{\mathrm{cl}}$ represents the radius of the cluster, and $-50 \leq\left[\mu_{\alpha} \cos \delta, \mu_{\delta}\right] \leq 50$. Based on these values, we defined the boundary of the proper motion. Fig. 2 shows vector point diagrams of the proper motion for the five clusters. Most stars in each cluster are distributed near the zero point $(0,0)$, but the average values of the proper motions are slightly off from the zero point. Some stars located far from this group may not be members of the cluster. We use an ellipse for defining the boundary, because dispersions along the RA-direction and the Dec-direction are not the same. This ellipse has an average of proper motions as a center, and 3 times of dispersions as axis lengths.

\subsection{Determining the Center and Size of the Clusters}

We determined the center of the clusters by finding the maximum surface number density of the member stars in each cluster. We investigated the peak value of the number density using the stars within $14 \leq r \leq 20$ for the clusters except for Pfleiderer 3 . The stars in Pfleiderer 3 are fainter than those in other clusters due to which we used stars within $18 \leq r \leq 20$. A searching bin used for finding the maximum is a circle of $R=0^{\prime} .25$ radius. If there are more than one local maximum of the number density, we moved the center of clusters to the position of a peak value which is confirmed visually. Using these new centers, we derived radial number density profiles of the clusters, as shown in Fig. 3. We determined visually the size of the clusters from the region beyond which the excess of the number density is indistinguishable from the outer region. The coordinates and sizes of the clusters from DAML02 and from this study are listed in Table 3. 

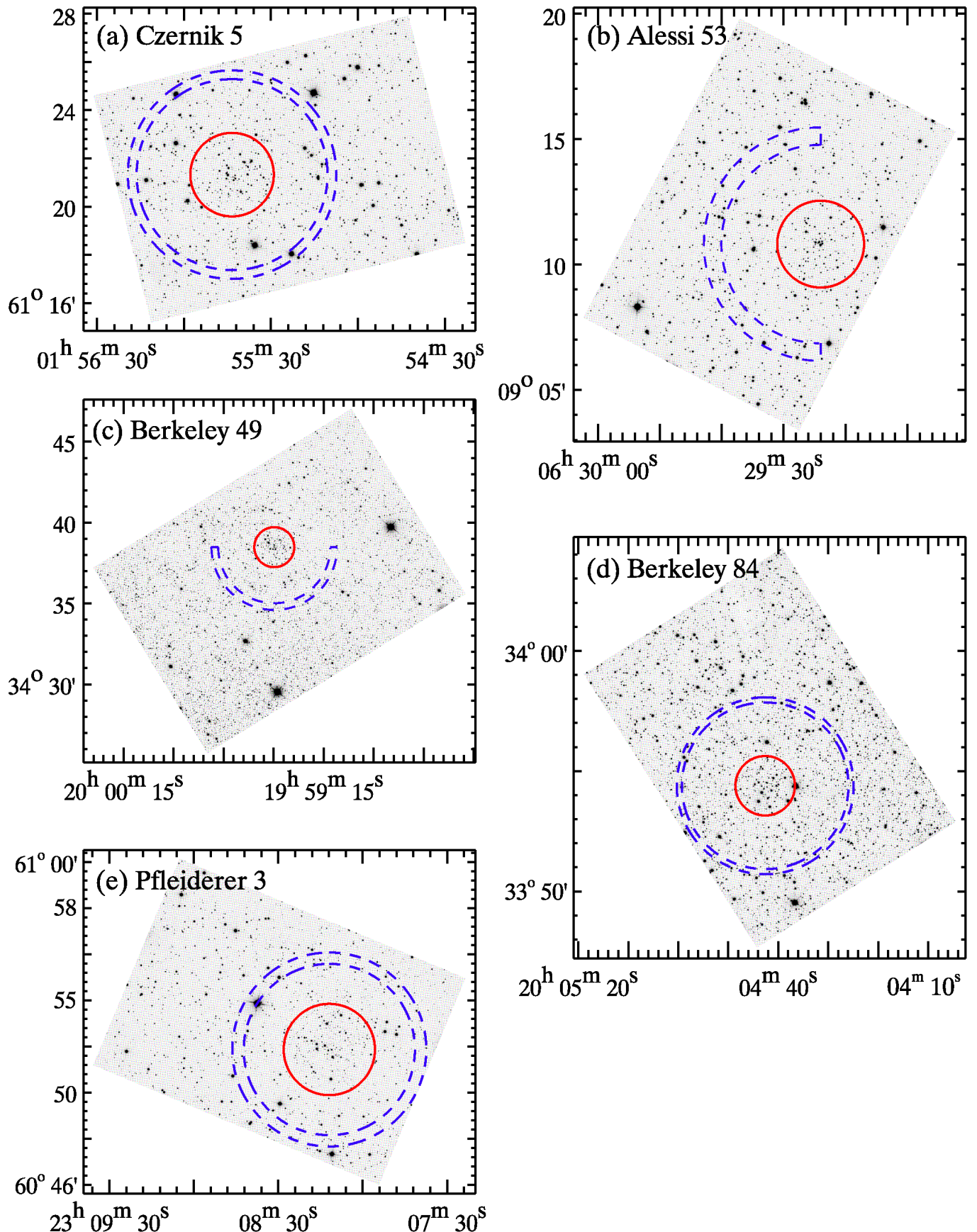

Fig. 1.- Gray-scale maps of the $r$ band images for target clusters which are obtained from the SDSS DAS. Images are rotated and combined using SWarp (Bertin et al. 2002). The x-axis and y-axis denote the RA and Dec coordinate, respectively. Each solid circle indicates the size of the cluster, and the region within dashed circles represents a background region which has the same area as that of the cluster region. The name of clusters and the radius of the inner circle or arc in each panel are as follows: (a) Czernik $5\left(R_{i n}=4^{\prime}\right)$, (b) Alessi $53\left(R_{i n}=4^{\prime}\right)$, (c) Berkeley $49\left(R_{i n}=3^{\prime} .5\right)$, (d) Berkeley $84\left(R_{i n}=3^{\prime} .5\right)$, and (e) Pfleiderer $3\left(R_{i n}=3^{\prime} .75\right)$. 
Table 1.

Transformation coefficients

\begin{tabular}{|c|c|c|c|c|c|c|c|c|c|}
\hline & \multicolumn{3}{|c|}{ Czernik 5} & \multicolumn{3}{|c|}{ Alessi 53} & \multicolumn{3}{|c|}{ Berkeley 49 (Field 1) } \\
\hline Filter & $\mathrm{Z}$ & $\mathrm{rms}$ & \# of stars & $\mathrm{Z}$ & $\mathrm{rms}$ & \# of stars & $\mathrm{Z}$ & $\mathrm{rms}$ & \# of stars \\
\hline $\mathrm{u}^{\prime}$ & 2.232 & 0.004 & 96 & 1.966 & 0.002 & 141 & 2.505 & 0.003 & 70 \\
\hline $\mathrm{g}^{\prime}$ & 1.074 & 0.001 & 258 & 1.160 & 0.002 & 309 & 1.067 & 0.001 & 84 \\
\hline $\mathrm{r}^{\prime}$ & 1.385 & 0.001 & 444 & 1.374 & 0.000 & 448 & 1.427 & 0.001 & 118 \\
\hline $\mathrm{i}^{\prime}$ & 1.764 & 0.001 & 618 & 1.697 & 0.001 & 635 & 1.693 & 0.003 & 130 \\
\hline $\mathrm{z}^{\prime}$ & 3.460 & 0.000 & 626 & 3.001 & 0.001 & 673 & 3.409 & 0.002 & 130 \\
\hline & \multicolumn{3}{|c|}{ Berkeley 49 (Field 2) } & \multicolumn{3}{|c|}{ Berkeley 84} & \multicolumn{3}{|c|}{ Pfleiderer 3} \\
\hline Filter & $\mathrm{Z}$ & $\mathrm{rms}$ & \# of stars & $\mathrm{Z}$ & $\mathrm{rms}$ & \# of stars & $\mathrm{Z}$ & $\mathrm{rms}$ & \# of stars \\
\hline $\mathrm{u}^{\prime}$ & 2.495 & 0.003 & 40 & 2.459 & 0.003 & 37 & 1.899 & 0.002 & 105 \\
\hline $\mathrm{g}^{\prime}$ & 1.069 & 0.002 & 44 & 1.055 & 0.002 & 45 & 1.094 & 0.001 & 219 \\
\hline $\mathrm{r}^{\prime}$ & 1.406 & 0.002 & 51 & 1.396 & 0.001 & 56 & 1.340 & 0.001 & 329 \\
\hline $\mathrm{i}^{\prime}$ & 1.637 & 0.003 & 57 & 1.738 & 0.002 & 54 & 1.710 & 0.001 & 427 \\
\hline $\mathrm{z}^{\prime}$ & 3.413 & 0.004 & 61 & 3.373 & 0.004 & 59 & 3.039 & 0.001 & 477 \\
\hline
\end{tabular}

Table 2.

ugriz Photometry of five open clusters ${ }^{\mathrm{a}}$

\begin{tabular}{|c|c|c|c|c|c|c|c|c|c|c|c|c|c|}
\hline ID & $\mathrm{RA}(\mathrm{J} 2000)$ & $\mathrm{DEC}(\mathrm{J} 2000)$ & $u$ & $\epsilon_{u}$ & $g$ & $\epsilon_{g}$ & $r$ & $\epsilon_{r}$ & $i$ & $\epsilon_{i}$ & $z$ & $\epsilon_{z}$ & Membership $^{\mathrm{b}}$ \\
\hline \multicolumn{14}{|c|}{ (a) Czernik 5} \\
\hline 7 & $01: 54: 25.22$ & $+61: 19: 46.2$ & 20.011 & 0.041 & 18.236 & 0.005 & 17.200 & 0.005 & 17.498 & 0.111 & & & 1 \\
\hline 185 & $01: 54: 35.82$ & $+61: 20: 06.3$ & 18.864 & 0.015 & 16.744 & 0.003 & 15.667 & 0.004 & 15.167 & 0.003 & 14.882 & 0.004 & 1 \\
\hline 326 & $01: 54: 40.78$ & $+61: 25: 14.2$ & 18.463 & 0.011 & 16.423 & 0.002 & 15.701 & 0.004 & 15.315 & 0.002 & 15.024 & 0.004 & 1 \\
\hline 421 & $01: 54: 43.31$ & $+61: 19: 26.3$ & 19.347 & 0.019 & 17.173 & 0.004 & 16.212 & 0.004 & 15.693 & 0.007 & 15.230 & 0.004 & 1 \\
\hline 440 & $01: 54: 43.76$ & $+61: 18: 46.1$ & 17.557 & 0.008 & 15.944 & 0.003 & 15.086 & 0.004 & 14.665 & 0.004 & 14.434 & 0.003 & 1 \\
\hline \multicolumn{14}{|c|}{ (b) Alessi 53} \\
\hline 2 & $06: 29: 03.20$ & $+09: 15: 26.0$ & 18.764 & 0.022 & 17.228 & 0.007 & 16.504 & 0.014 & 16.084 & 0.003 & 15.850 & 0.008 & 1 \\
\hline 129 & $06: 29: 08.27$ & $+09: 14: 00.6$ & 21.990 & 0.165 & 19.872 & 0.011 & 18.901 & 0.013 & 18.297 & 0.005 & 17.925 & 0.019 & 1 \\
\hline 217 & $06: 29: 10.81$ & $+09: 12: 16.3$ & - & - & 21.939 & 0.062 & 20.615 & 0.042 & 19.825 & 0.020 & 19.463 & 0.056 & 2 \\
\hline 262 & $06: 29: 11.74$ & $+09: 13: 18.9$ & 19.821 & 0.032 & 18.043 & 0.004 & 17.235 & 0.007 & 16.876 & 0.012 & 16.625 & 0.009 & 1 \\
\hline 350 & $06: 29: 13.48$ & $+09: 13: 43.4$ & 18.472 & 0.014 & 16.939 & 0.003 & 16.252 & 0.006 & 15.958 & 0.009 & 15.743 & 0.006 & 1 \\
\hline \multicolumn{14}{|c|}{ (c) Berkeley 49} \\
\hline 114 & $19: 58: 36.92$ & $+34: 36: 30.3$ & 21.338 & 0.190 & 18.870 & 0.007 & 17.724 & 0.008 & 17.124 & 0.012 & 16.611 & 0.010 & 1 \\
\hline 262 & $19: 58: 39.12$ & $+34: 36: 10.3$ & 18.513 & 0.022 & 16.573 & 0.003 & 15.602 & 0.006 & 15.162 & 0.008 & 14.873 & 0.005 & 1 \\
\hline 692 & $19: 58: 43.06$ & $+34: 37: 09.3$ & - & - & 22.495 & 0.070 & 20.740 & 0.033 & 19.974 & 0.030 & 19.249 & 0.040 & 1 \\
\hline 810 & $19: 58: 43.94$ & $+34: 38: 52.2$ & 17.551 & 0.015 & 15.771 & 0.003 & 14.886 & 0.006 & 14.492 & 0.010 & 14.262 & 0.003 & 1 \\
\hline 1006 & $19: 58: 45.45$ & $+34: 33: 57.5$ & - & - & 21.399 & 0.029 & 19.680 & 0.014 & 18.776 & 0.012 & 18.104 & 0.016 & 1 \\
\hline \multicolumn{14}{|c|}{ (d) Berkeley 84} \\
\hline 48 & $20: 04: 06.70$ & $+33: 53: 18.6$ & 19.770 & 0.033 & 18.008 & 0.006 & 17.114 & 0.004 & 16.533 & 0.006 & 16.316 & 0.008 & 1 \\
\hline 104 & 20:04:07.70 & $+33: 53: 13.6$ & 17.634 & 0.011 & 16.066 & 0.002 & 15.049 & 0.004 & 14.356 & 0.005 & 14.003 & 0.006 & 1 \\
\hline 236 & $20: 04: 09.33$ & $+33: 52: 46.1$ & 16.651 & 0.007 & 15.400 & 0.004 & 14.993 & 0.003 & 14.698 & 0.003 & 14.725 & 0.006 & 1 \\
\hline 331 & $20: 04: 10.49$ & $+33: 54: 43.3$ & 18.273 & 0.014 & 16.776 & 0.003 & 16.174 & 0.006 & 15.816 & 0.005 & 15.767 & 0.007 & 1 \\
\hline 509 & $20: 04: 11.92$ & $+33: 52: 28.4$ & 18.187 & 0.011 & 16.401 & 0.019 & 16.002 & 0.006 & 15.702 & 0.015 & 15.663 & 0.016 & 1 \\
\hline \multicolumn{14}{|c|}{ (e) Pfleiderer 3} \\
\hline 72 & $23: 07: 32.16$ & $+60: 52: 32.3$ & - & - & 21.408 & 0.046 & 19.630 & 0.020 & 18.785 & 0.012 & 18.240 & 0.014 & 2 \\
\hline 108 & $23: 07: 35.17$ & $+60: 55: 21.9$ & 19.516 & 0.025 & 17.402 & 0.006 & 16.276 & 0.006 & 15.690 & 0.007 & 15.275 & 0.011 & 1 \\
\hline 216 & $23: 07: 40.73$ & $+60: 53: 33.9$ & 19.588 & 0.021 & 16.382 & 0.004 & 14.788 & 0.004 & 14.030 & 0.004 & 13.473 & 0.003 & 1 \\
\hline 285 & $23: 07: 42.75$ & $+60: 49: 28.7$ & 17.736 & 0.007 & 16.045 & 0.009 & 15.356 & 0.003 & 15.072 & 0.003 & 14.811 & 0.006 & 1 \\
\hline 372 & $23: 07: 45.70$ & $+60: 48: 35.5$ & 21.406 & 0.090 & 19.337 & 0.007 & 18.014 & 0.007 & 17.385 & 0.008 & 16.962 & 0.010 & 1 \\
\hline
\end{tabular}

a This is a sample of the full tables, which are compiled from the catalog of each cluster. The full tables will be available from the authors upon request.

${ }^{\mathrm{b}} 1$ and 2 represent cluster members and non-members, respectively. 


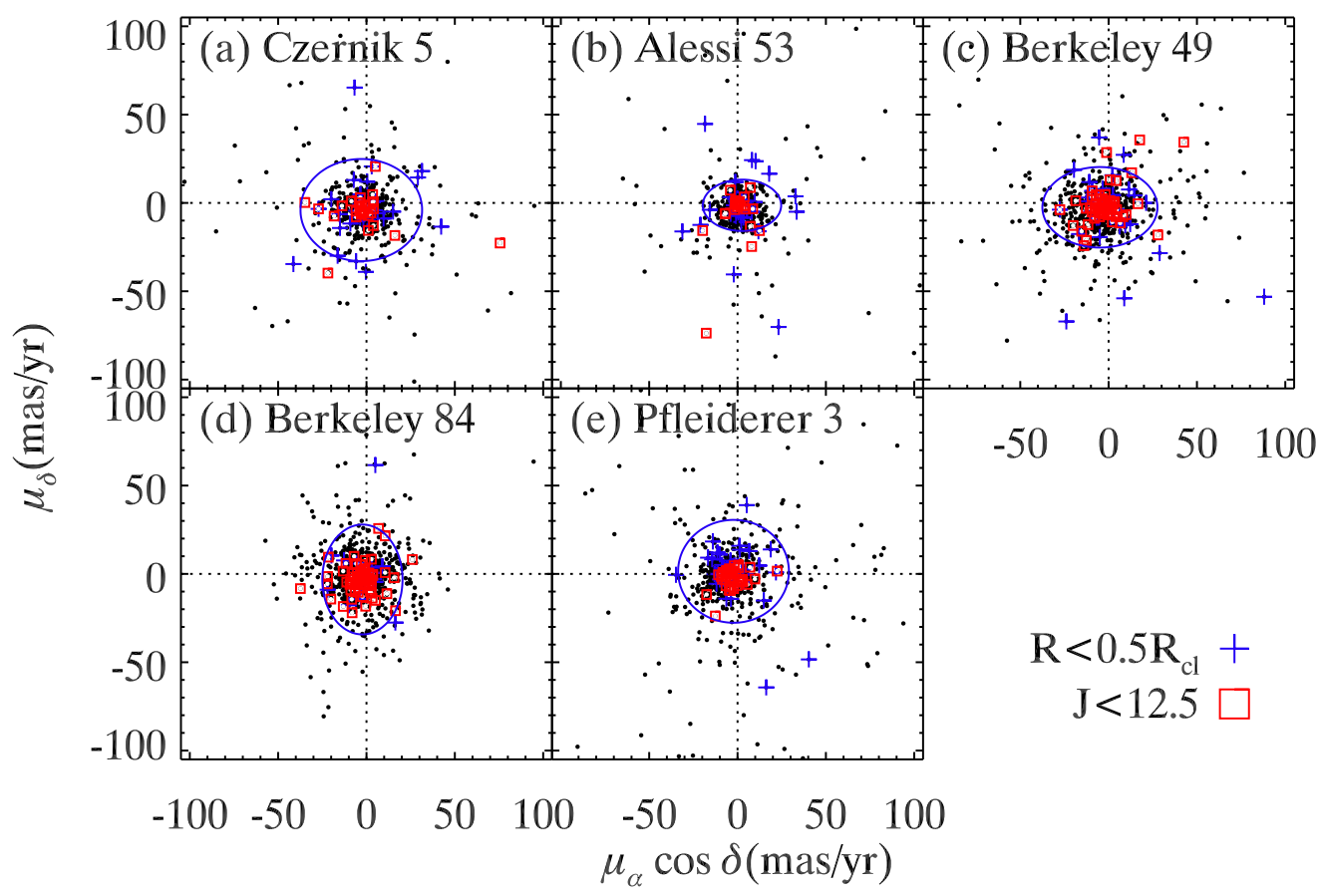

Fig. 2. - Vector point diagrams of the proper motions in the field of each cluster: (a) Czernik 5, (b) Alessi 53, (c) Berkeley 49, (d) Berkeley 84 and (e) Pfleiderer 3. The ellipses around the zero point are the boundary to exclude non-member stars. Dots represent the stars with $\mathrm{J}<14$, squares represent the bright stars with $\mathrm{J}<12.5$ and plus symbols represent the stars inside the half radius of the cluster. These symbols indicate that they are more probable members than other stars of the cluster, but some of them are lying outside of the ellipse.

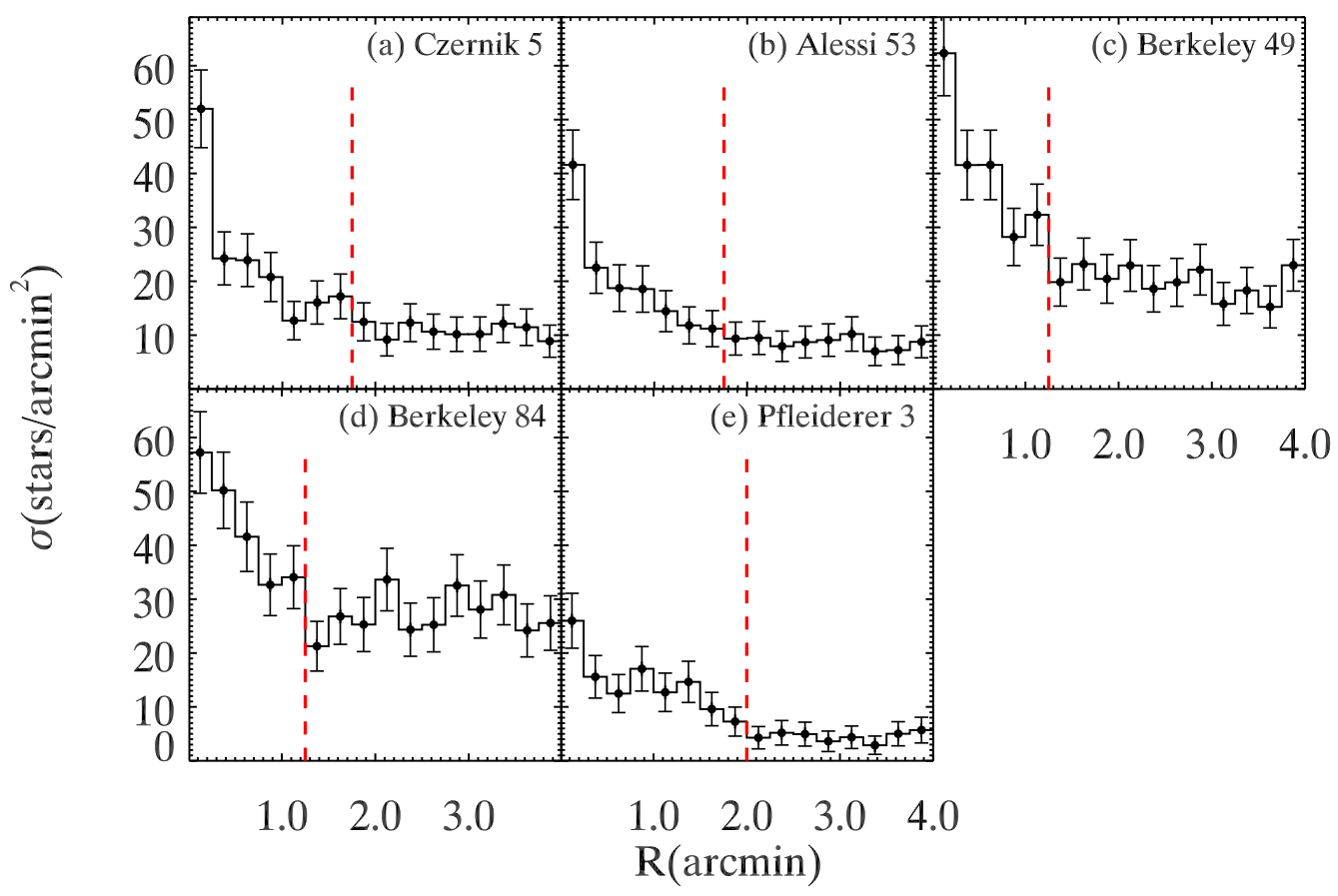

Fig. 3.- Radial number density profiles for the stars in the clusters: (a) Czernik 5, (b) Alessi 53, (c) Berkeley 49, (d) Berkeley 84, and (e) Pfleiderer 3. The dashed lines represent the size of each cluster. Error bars denote Poisson errors. 
Table 3.

The coordinates and sizes of the target clusters

\begin{tabular}{|c|c|c|c|c|c|c|c|c|c|}
\hline \multirow[b]{2}{*}{ Name } & \multicolumn{3}{|c|}{ DAML02 } & \multicolumn{3}{|c|}{ This study } & \multicolumn{3}{|c|}{ (This study) - (DAML02) } \\
\hline & $\begin{array}{c}\mathrm{RA} \\
(\mathrm{h} \mathrm{m} \mathrm{s})\end{array}$ & $\begin{array}{c}\text { DEC } \\
\left(\begin{array}{lll}0 & \prime & \prime \prime\end{array}\right)\end{array}$ & $\begin{array}{c}R_{\mathrm{cl}} \\
\left(^{\prime}\right)\end{array}$ & $\begin{array}{c}\mathrm{RA} \\
(\mathrm{h} \mathrm{m} \mathrm{s})\end{array}$ & $\begin{array}{c}\text { DEC } \\
\left({ }^{\circ} \prime \prime \prime \prime\right)\end{array}$ & $\begin{array}{l}R_{\mathrm{cl}} \\
\left(^{\prime}\right)\end{array}$ & $\Delta \mathrm{RA}$ & $\triangle \mathrm{DEC}$ & $\Delta R_{\mathrm{cl}}$ \\
\hline Czerr & 015506 & +612000 & 0.75 & 015543.4 & +612121 & 1.75 & $561^{\prime \prime} .0$ & $81^{\prime \prime}$ & $1^{\prime} .00$ \\
\hline Alessi 53 & 062924 & +091055 & 1.4 & 062924.0 & +091049 & 1.75 & $0^{\prime \prime} .0$ & $-6^{\prime \prime}$ & $0^{\prime} .35$ \\
\hline Berkeley 49 & 195931 & +343848 & 2.0 & 195929.8 & +343830 & 1.25 & $-18^{\prime \prime} .0$ & $-18^{\prime \prime}$ & $-0^{\prime} .75$ \\
\hline Berkeley 84 & 200443 & +335418 & 4.5 & 200442.6 & +335424 & 1.25 & $-6^{\prime \prime} .0$ & $6^{\prime \prime}$ & $-3^{\prime} .25$ \\
\hline Pfleiderer 3 & 230811 & +605224 & 2.0 & 230811.8 & +605154 & 2.00 & $12^{\prime \prime} .0$ & $-30^{\prime \prime}$ & $0^{\prime} .00$ \\
\hline
\end{tabular}

\subsection{Determining the Physical Parameters}

We derived the physical parameters of these clusters using the following methods. We determined the foreground reddening using the Padova isochrones (Girardi et al. 2004) in the CMD and the Zero Age Main Sequence (ZAMS) in the Color-Color Diagram (CCD). The extinction law in An et al. (2008) is used for determining reddenings: $A_{u} / A_{V}=1.574, A_{g} / A_{V}=1.189$, $A_{r} / A_{V}=0.877, A_{i} / A_{V}=0.673$, and $A_{z} / A_{V}=0.489$, assuming $R_{V} \equiv A_{V} / E(B-V)=3.1$. After determining the foreground reddening, we estimated the distance and age for the clusters using the isochrone fitting in the CMD. For this fitting, we need to know the metallicity of the clusters. However, it is not easy to derive metallicity from photometry. Therefore we adopted the metallicity for the clusters using the relation between $[\mathrm{Fe} / \mathrm{H}]$ and the galactocentric distance $R_{\mathrm{GC}}$.

Fig. 4 displays $[\mathrm{Fe} / \mathrm{H}]$ and $R_{\mathrm{GC}}$ relation based on data for 186 clusters with $[\mathrm{Fe} / \mathrm{H}]$ values in DAML02. We derived the mean metallicity for each $1 \mathrm{kpc}-$ size bin as a function of $R_{\mathrm{GC}}$, as plotted in the same figure. The mean metallicity decreases as $R_{\mathrm{GC}}$ increases for $R_{\mathrm{GC}} \lesssim 12 \mathrm{kpc}$, and appears to be constant at $[\mathrm{Fe} / \mathrm{H}]$ $\approx-0.3$ and thereafter. We fitted the data for $R_{\mathrm{GC}} \lesssim$ $12 \mathrm{kpc}$ with a linear function, obtaining $<[\mathrm{Fe} / \mathrm{H}]>=$ $(-0.076 \pm 0.013) R_{\mathrm{GC}}+(0.600 \pm 0.116)$ with $\mathrm{rms}=0.029$. This value for the radial gradient is similar to previous estimates (Friel et al. 2002; Chen et al. 2003; Andreuzzi et al. 2011). We adopt the metallicity for each cluster using this relation: $[\mathrm{Fe} / \mathrm{H}]=+0.022,+0.023,-0.164$, 0.203 , and $-0.344(\mathrm{Z}=0.019,0.019,0.016,0.016,0.013)$ for Berkeley 49, Berkeley 84, Czernik 5, Pleiderer 3, and Alessi 53, respectively.

\section{RESULTS}

We describe the results we obtained applying the above methods for each cluster in the following.

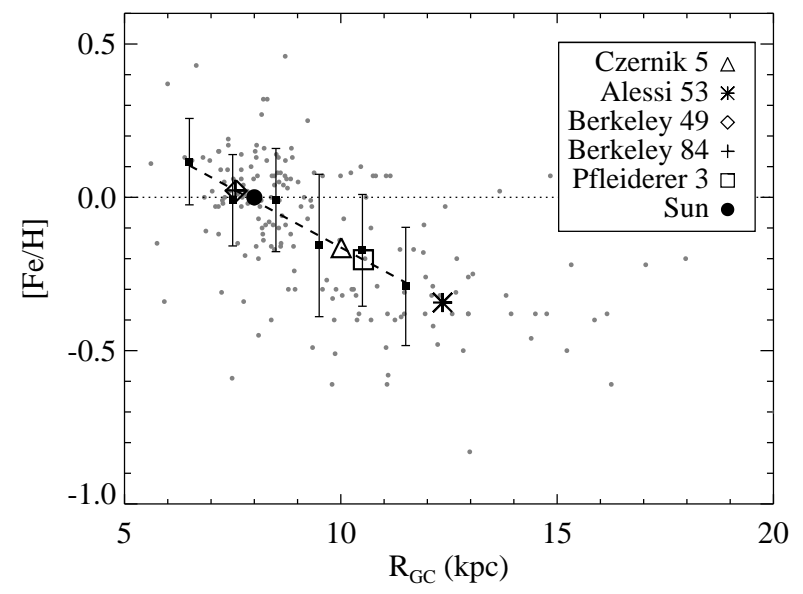

Fig. 4.- Metallicity vs. Galactocentric distance diagram for 186 OCs in DAML02. Filled squares and error bars represent the mean metallicities and the standard deviations. Large symbols represent the assumed metallicity of the target clusters in this study.

\subsection{Czernik 5}

The coordinates for the center of Czernik 5 that we derived are ( $\left.\alpha=01 \mathrm{~h} 55 \mathrm{~m} \mathrm{43.4s,} \delta=+61^{\circ} 21^{\prime} 21^{\prime \prime}\right)$, which are different by $561^{\prime \prime} .0$ in RA and $81^{\prime \prime}$ in Dec from the values in DAML02. The radial number density profile of Czernik 5 derived using the new cluster center is shown in Fig. 3a. From this figure, the radius of Czernik 5 is determined to be $R_{\mathrm{cl}}=1^{\prime} .75 \pm 0.13$.

Fig. 5 shows (a) an $r-(g-r)$ CMD of Czernik 5 , and (b) a CMD of the background region which has the same area as that of the cluster region, as shown in Fig. 1a. Main sequences are found on both CMDs, but more stars are seen in the cluster's CMD. It is notable that the cluster CMD shows about two dozen bright stars at $0.8 \leq(g-r) \leq 1.2$ and $14 \leq r \leq 17$, while the background CMD shows only a few. Most of these stars are considered to be members of the cluster. About half of these bright stars are located close to the cluster center, within the half of the radius. 


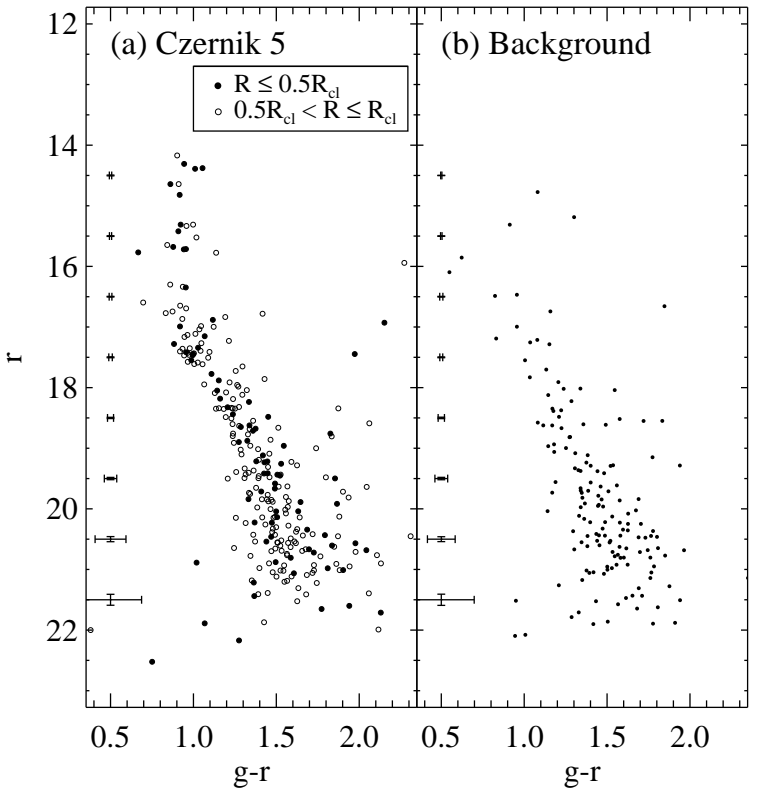

Fig. 5.- (a) $r-(g-r)$ CMD of the stars in Czernik 5. Open circles are stars inside the radius of Czernik 5 and filled dots are those inside the half radius of the cluster. (b) $r-(g-r)$ CMD of the stars in the background region, as shown in Fig. 1a. Mean photometric errors are marked on the left side of each panel.

Fig. 6 shows the result of the isochrone fitting. We derived its age, $\log ($ age $[\mathrm{yr}])=8.45$, and the reddenings: $E(B-V)=1.20 \pm 0.05,1.20 \pm 0.05,1.15 \pm 0.05$, and $0.85 \pm 0.05$ from $r-(g-r) \mathrm{CMD}, r-(g-i) \mathrm{CMD}$, $r-(g-z)$ CMD and $r-(u-g)$ CMD, respectively. The corresponding distance moduli we derived are $(m-$ $M)_{0}=12.2 \pm 0.2$ for all CMDs. These values are listed in Table 4. The reddening values derived from the first two CMDs agree, while the latter two have somewhat smaller values. This trend is similarly seen in other clusters. This indicates that the theoretical colors in the Padova isochrones, $(g-z)$ and $(u-g)$ are too red by $\sim 0.1$. Hereafter, we adopt the values for the reddening and distance modulus derived from $r-(g-r)$ CMD for further analysis, because this CMD is considered to be most reliable for cluster analysis (An et al. 2008). Fig. 6b and 6c display the $(u-g)-(g-r)$ CCD and $(g-r)-(r-i)$ CCD for the bright main sequence stars located within the solid box in Fig. 6a. They are well fitted by the ZAMS shifted according to the reddening $E(B-V)=1.20$.

\subsection{Alessi 53}

This cluster is not easy to discern in the gray-scale map of $r$ band image (Fig. 1b). However, the radial number density profile of the stars around this cluster shows clearly an excess in the cluster region. The coor-
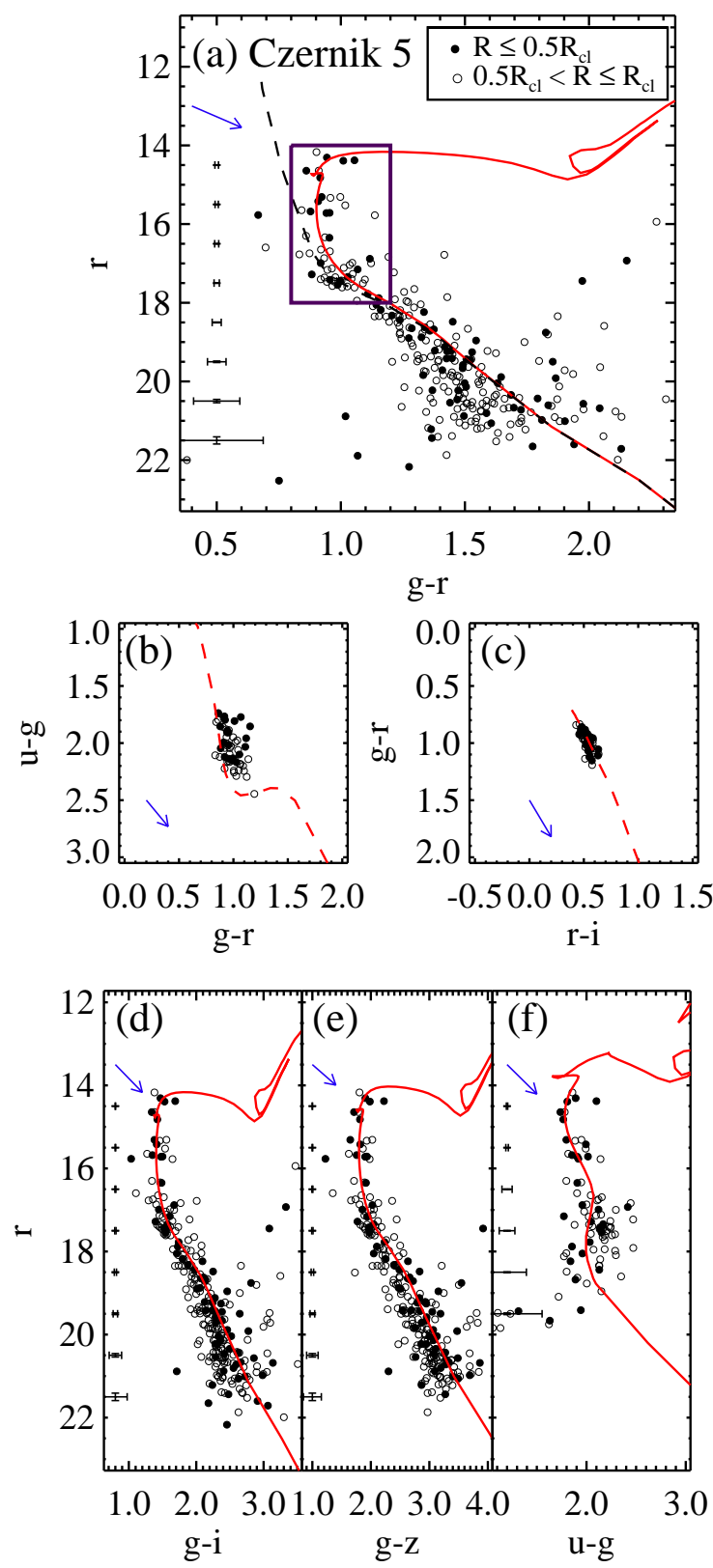

Fig. 6. - CMDs and CCDs of Czernik 5. Symbols represent the same as in Fig. 5. The solid line represents an isochrone for $\log ($ age $[\mathrm{yr}])=8.45$ and $Z=0.016$, and the dashed line represents the ZAMS. The arrow in each panel represents a reddening vector. (a) Isochrone fitting for $\mathrm{Cz}-$ ernik 5 in the $r-(g-r)$ CMD. The isochrone is shifted according to $E(B-V)=1.20$ and $(m-M)_{0}=12.2$. The solid box contains main sequence stars that are plotted in the following CCDs. (b) $(u-g)-(g-r)$ CCD. (c) $(g-r)-(r-i)$ CCD. (d) $r-(g-i)$ CMD. (e) $r-(g-z)$ CMD. (f) $r-(u-g)$ CMD. 
dinates for the center of this cluster $(\alpha=06 \mathrm{~h} 29 \mathrm{~m} 24.0 \mathrm{~s}$, $\delta=+09^{\circ} 10^{\prime} 49^{\prime \prime}$ ) are different by $-6^{\prime \prime}$ in Dec from the value in DAML02. Fig. 3b displays the radial number density profile of this cluster we derived. We determined the radius of Alessi 53 to be $R_{\mathrm{cl}}=1^{\prime} .75 \pm 0.13$.

The CMD of Alessi 53 in Fig. 7 shows a narrow main sequence at $16 \leq r \leq 18.5$ which is not seen in the CMD of background stars. There is seen a hint of another sequence that is located $\sim 0.8 \mathrm{mag}$ above the main sequence. This may be a binary sequence of this cluster.

Fig. 8 shows the result of the isochrone fitting. We derived its age, $\log ($ age $[\mathrm{yr}])=8.4$, and the reddenings: $E(B-V)=0.80 \pm 0.05,0.80 \pm 0.05,0.75 \pm 0.05$, and $0.70 \pm 0.05$ from $r-(g-r)$ CMD, $r-(g-i)$ CMD, $r-(g-z)$ CMD, and $r-(u-g)$ CMD, respectively. The corresponding distance moduli we derived are $(m-$ $M)_{0}=13.3 \pm 0.15$ for $r-(g-r)$ CMD and $r-(g-i)$ CMD, and $(m-M)_{0}=13.5 \pm 0.15$ for $r-(g-z)$ CMD and $r-(u-g)$ CMD. These values are listed in Table 5. The reddening values derived from the first two CMDs agree, while the latter two have somewhat smaller values. Fig. $8 \mathrm{~b}$ and $8 \mathrm{c}$ display the $(u-g)-(g-$ $r)$ CCD and $(g-r)-(r-i)$ CCD for the bright main sequence stars located within the solid box in Fig. 8a. They are well fitted by the ZAMS shifted according to the reddening $E(B-V)=0.80$.

\subsection{Berkeley 49}

The coordinates for the center of Berkeley $49(\alpha=$ 19h $59 \mathrm{~m} 29.8 \mathrm{~s}, \delta=+34^{\circ} 38^{\prime} 30^{\prime \prime}$ ) derived in this study are different by $-18^{\prime \prime} .0$ in RA and $-18^{\prime \prime}$ in Dec from the values in DAML02. From the radial number density profile in Fig. 3c, we determined the radius of Berkeley 49 to be $R_{\mathrm{cl}}=1^{\prime} .25 \pm 0.13$.

The CMD of this cluster in Fig. 9 shows a rather broad main sequence. This may be due to severe foreground reddening. The bright main sequence in the cluster CMD is not clearly seen in the background CMD so that most of the stars in this sequence are probably the cluster members. The main sequence turnoff point appears to be located at $(g-r) \sim 1.1$ and $r \sim 16.5$.

Table 4.

Estimates for reddenings and distance moduli of Czernik 5 based on different colors

\begin{tabular}{ccc}
\hline \hline CMD & $E(B-V)$ & $(m-M)_{0}$ \\
\hline$r-(g-r)$ & $1.20 \pm 0.05$ & $12.2 \pm 0.2$ \\
$r-(g-i)$ & $1.20 \pm 0.05$ & $12.2 \pm 0.2$ \\
$r-(g-z)$ & $1.15 \pm 0.05$ & $12.2 \pm 0.2$ \\
$r-(u-g)$ & $0.85 \pm 0.05$ & $12.2 \pm 0.2$ \\
\hline
\end{tabular}

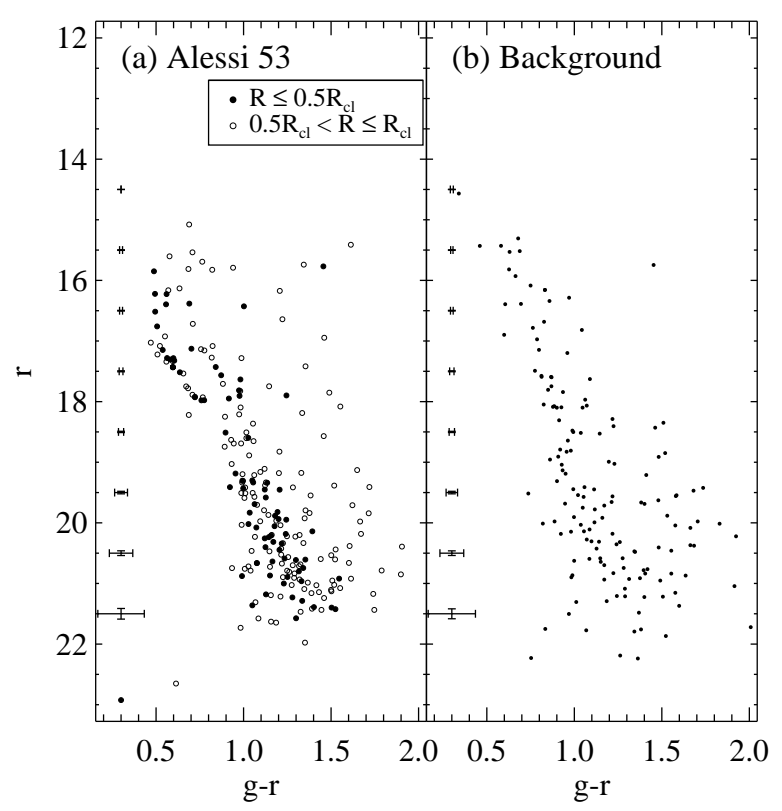

Fig. 7.- (a) $r-(g-r)$ CMD of the stars in Alessi 53. Open circles are stars inside the radius of Alessi 53 and filled dots are those inside the half radius of the cluster. (b) $r-(g-r)$ CMD of stars in the background region which is shown in Fig. 1b. Mean photometric errors are marked on the left side of each panel.

Fig. 10 shows the result of the isochrone fitting. We derived its age, $\log ($ age $[y r])=8.9$, and derived the reddenings and distance moduli from CMDs with different colors: $E(B-V)=1.18 \pm 0.05$ and $(m-M)_{0}=$ $11.6 \pm 0.2$ from $r-(g-r)$ CMD, $E(B-V)=1.15 \pm 0.05$ and $(m-M)_{0}=11.6 \pm 0.2$ from $r-(g-i) \mathrm{CMD}$, $E(B-V)=1.10 \pm 0.05$ and $(m-M)_{0}=11.8 \pm 0.2$ from $r-(g-z) \mathrm{CMD}$, and $E(B-V)=1.05 \pm 0.05$ and $(m-M)_{0}=11.6 \pm 0.2$ from $r-(u-g)$ CMD. These values are also listed in Table 6 . The reddening values derived from the CMDs are decreasing from the $r-(g-r)$ CMD to the $r-(u-g)$ CMD. Fig. 10b and 10c display the $(u-g)-(g-r) \mathrm{CCD}$ and $(g-r)-(r-i)$

Table 5.

Estimates for reddenings and distance moduli of Alessi 53 based on different colors

\begin{tabular}{ccc}
\hline \hline $\mathrm{CMD}$ & $E(B-V)$ & $(m-M)_{0}$ \\
\hline$r-(g-r)$ & $0.80 \pm 0.05$ & $13.3 \pm 0.15$ \\
$r-(g-i)$ & $0.80 \pm 0.05$ & $13.3 \pm 0.15$ \\
$r-(g-z)$ & $0.75 \pm 0.05$ & $13.5 \pm 0.15$ \\
$r-(u-g)$ & $0.70 \pm 0.05$ & $13.5 \pm 0.15$ \\
\hline
\end{tabular}



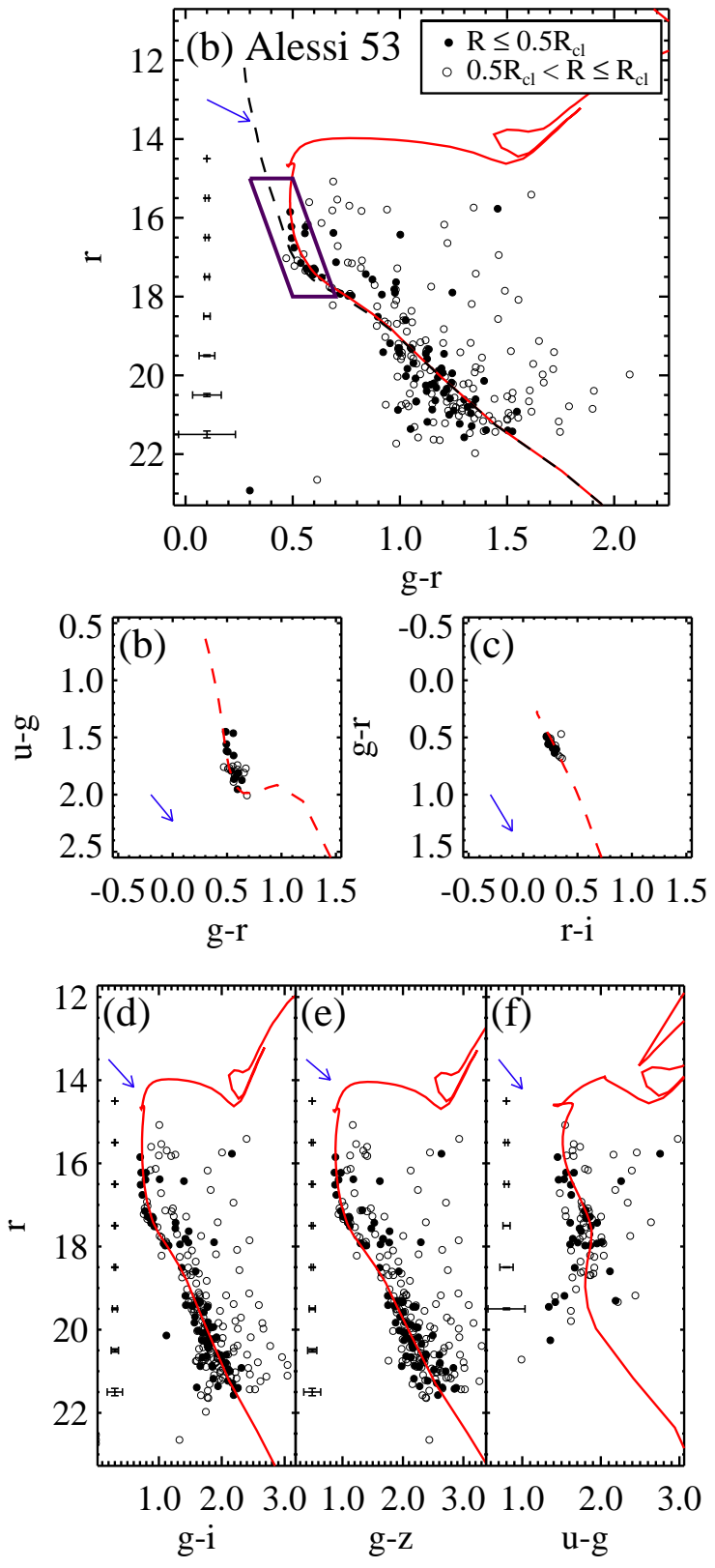

Fig. 8.- CMDs and CCDs of Alessi 53. Symbols represent the same as in Fig. 7. The solid line represents an isochrone for $\log (\operatorname{age}[\mathrm{yr}])=8.4$ and $Z=0.013$, and the dashed line represents the ZAMS. The arrow in each panel represents a reddening vector. (a) Isochrone fitting for Alessi 53. The isochrone is shifted according to $E(B-V)=0.80$ and $(m-$ $M)_{0}=13.3$. The inclined solid box contains main sequence stars which are plotted in the following CCDs. (b) $(u-g)-$ $(g-r)$ CCD. (c) $(g-r)-(r-i)$ CCD. (d) $r-(g-i)$ CMD. (e) $r-(g-z)$ CMD. (f) $r-(u-g)$ CMD.

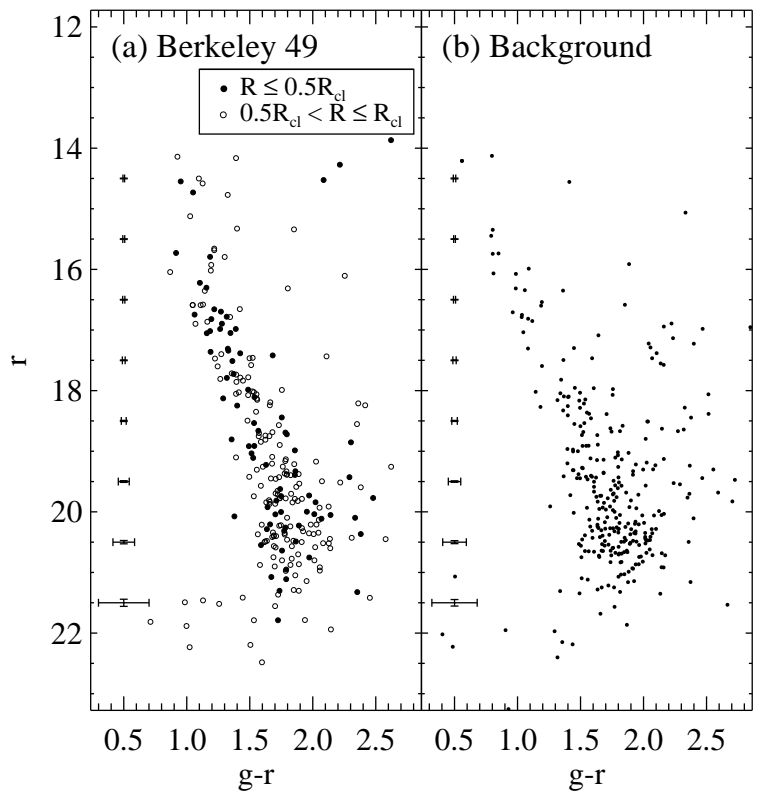

Fig. 9.- (a) $r-(g-r)$ CMD of the stars in Berkeley 49 . Open circles are stars inside the radius of Berkeley 49 and filled dots are stars inside the half radius of the cluster. (b) $r-(g-r)$ CMD of stars in the background region, which is shown in Fig. 1c. Mean photometric errors are marked on the left side of each panel.

CCD for the bright main sequence stars located within the upper solid box located within $16<r<18$ in Fig. 10a. They are well fitted by the ZAMS shifted according to the reddening $E(B-V)=1.18$.

\subsection{Berkeley 84}

The coordinates for the center of Berkeley $84(\alpha=$ $20 \mathrm{~h} 04 \mathrm{~m} 42.6 \mathrm{~s}, \delta=+33^{\circ} 54^{\prime} 24^{\prime \prime}$ ) derived in this study are different by $-6^{\prime \prime} .0$ in RA and $6^{\prime \prime}$ in Dec from the values in DAML02. Fig. 3d shows the radial number density profile of Berkeley 84. Based on the profile, we determined the radius of Berkeley 84 to be $R_{\mathrm{cl}}=$

Table 6.

Estimates for reddenings and distance moduli of Berkeley 49 with different colors

\begin{tabular}{ccc}
\hline \hline CMD & $E(B-V)$ & $(m-M)_{0}$ \\
\hline$r-(g-r)$ & $1.18 \pm 0.05$ & $11.6 \pm 0.2$ \\
$r-(g-i)$ & $1.15 \pm 0.05$ & $11.6 \pm 0.2$ \\
$r-(g-z)$ & $1.10 \pm 0.05$ & $11.8 \pm 0.2$ \\
$r-(u-g)$ & $1.05 \pm 0.05$ & $11.6 \pm 0.2$ \\
\hline
\end{tabular}



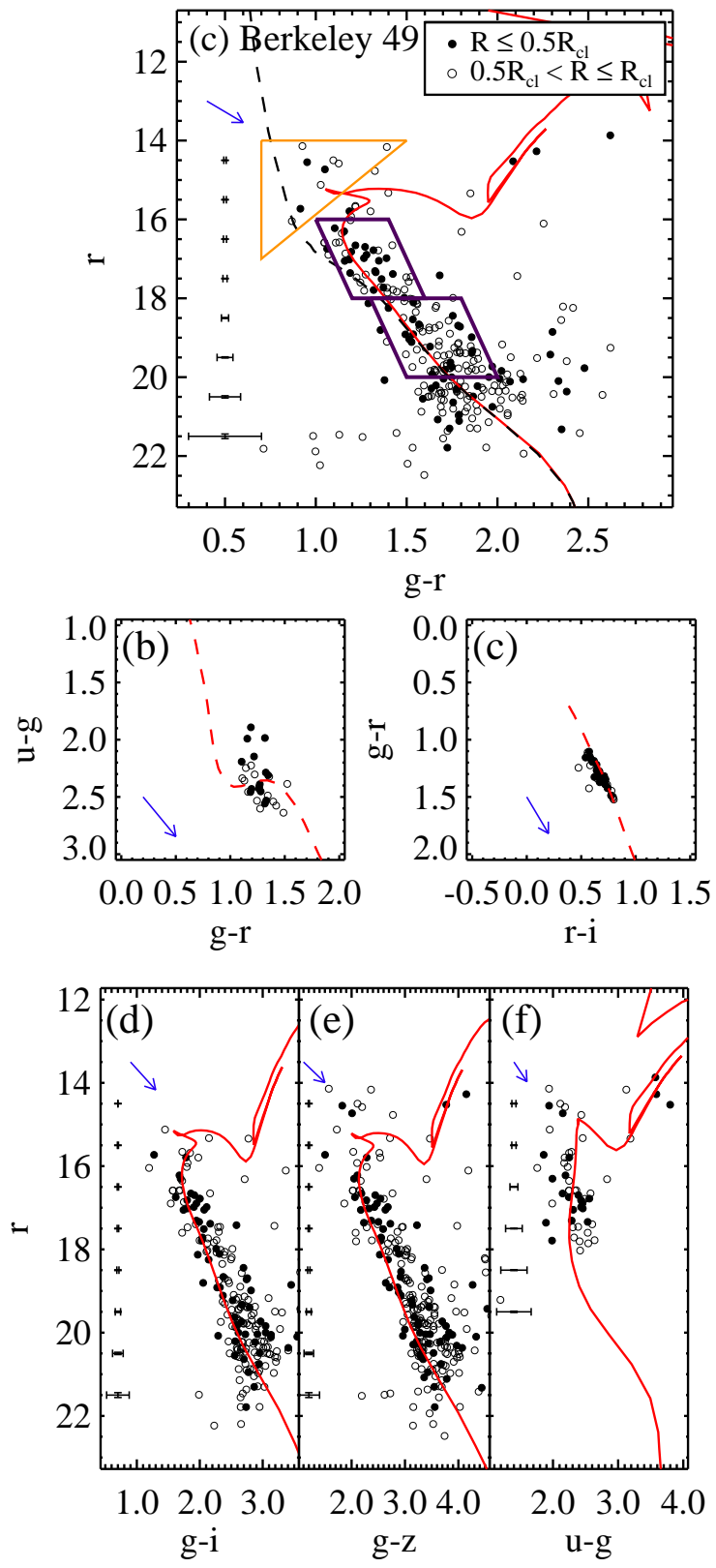

Fig. 10. - CMDs and CCDs of Berkeley 49. Symbols represent the same as in Fig. 9. The solid line represents an isochrone for $\log (\operatorname{age}[\mathrm{yr}])=8.9$ and $Z=0.019$, and the dashed line represents the ZAMS. The arrow in each panel represents a reddening vector. (a) The isochrone fitting for Berkeley 49. The isochrone is shifted according to $E(B-V)=1.18$ and $(m-M)_{0}=11.6$. The stars located within the triangle are probably the field stars. A parallelogram located within $16<r<18$ contains main sequence stars those are plotted in following CCDs. Another parallelogram, located within $18<r<20$, contains faint main sequence stars. Radial distributions of stars located within each region are discussed in Section 5.3. (b) $(u-g)-(g-r)$ CCD. (c) $(g-r)-(r-i)$ CCD. (d) $r-(g-i)$ CMD. (e) $r-(g-z)$ CMD. (f) $r-(u-g)$ CMD.

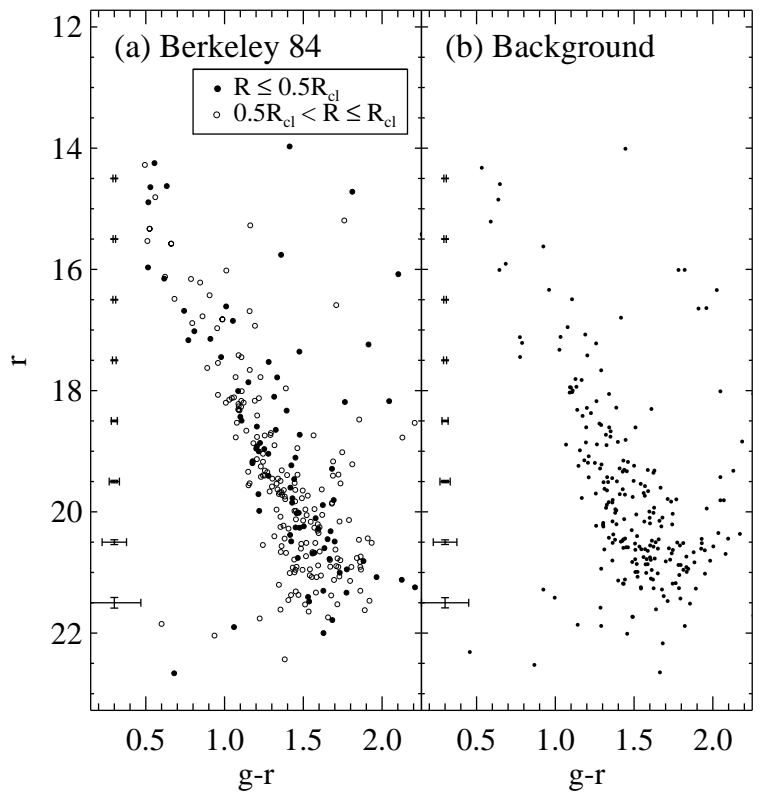

Fig. 11.- (a) $r-(g-r)$ CMD of the stars in Berkeley 84 . Open circles are stars inside the radius of Berkeley 84 and filled dots are stars inside the half radius of the cluster. (b) $r-(g-r)$ CMD of stars in the background region, which is shown in Fig. 1d. Mean photometric errors are marked on the left side of each panel.

$1^{\prime} .25 \pm 0.13$.

It is rather hard to study Berkeley 84 only using the SDSS because five stars inside the radius of Berkeley 84 are saturated. These bright stars $(r \leq 14)$ are not shown in CMDs and the main sequence of Berkeley 84 looks broad. Therefore, the CMDs of Berkeley 84 and the background region look similar (Fig. 11). This may be due to severe foreground reddening. In spite of that, the number and distribution of the stars at $(g-r) \simeq 0.5$ and $14 \leq r \leq 16$ look different from those of the background and most of the stars in the bright main sequence are probably the cluster members.

Fig. 12 shows the result of the isochrone fitting. We derived its age, $\log ($ age $[\mathrm{yr}])=8.65$, and the reddenings: $E(B-V)=0.73 \pm 0.06,0.80 \pm 0.06,0.73 \pm 0.06$ and $0.65 \pm 0.06$ from $r-(g-r)$ CMD,$r-(g-i)$ CMD, $r-(g-z)$ CMD and $r-(u-g)$ CMD, respectively. The corresponding distance moduli we derived are $(m-$ $M)_{0}=12.2 \pm 0.2$ for all CMDs. These values are also listed in Table 7 . The reddening values derived from the $r-(g-r)$ and $r-(g-z)$ CMDs agree, while the reddening from the $r-(g-i) \mathrm{CMD}$ is higher and that from the $r-(u-g)$ CMD is smaller. Fig. 12(b) and (c) display the $(u-g)-(g-r)$ CCD and $(g-r)-(r-i)$ CCD for the bright main sequence stars located within the solid box in Fig. 12a. They are well-fitted by the ZAMS shifted according to the reddening $E(B-V)=0.73$. 

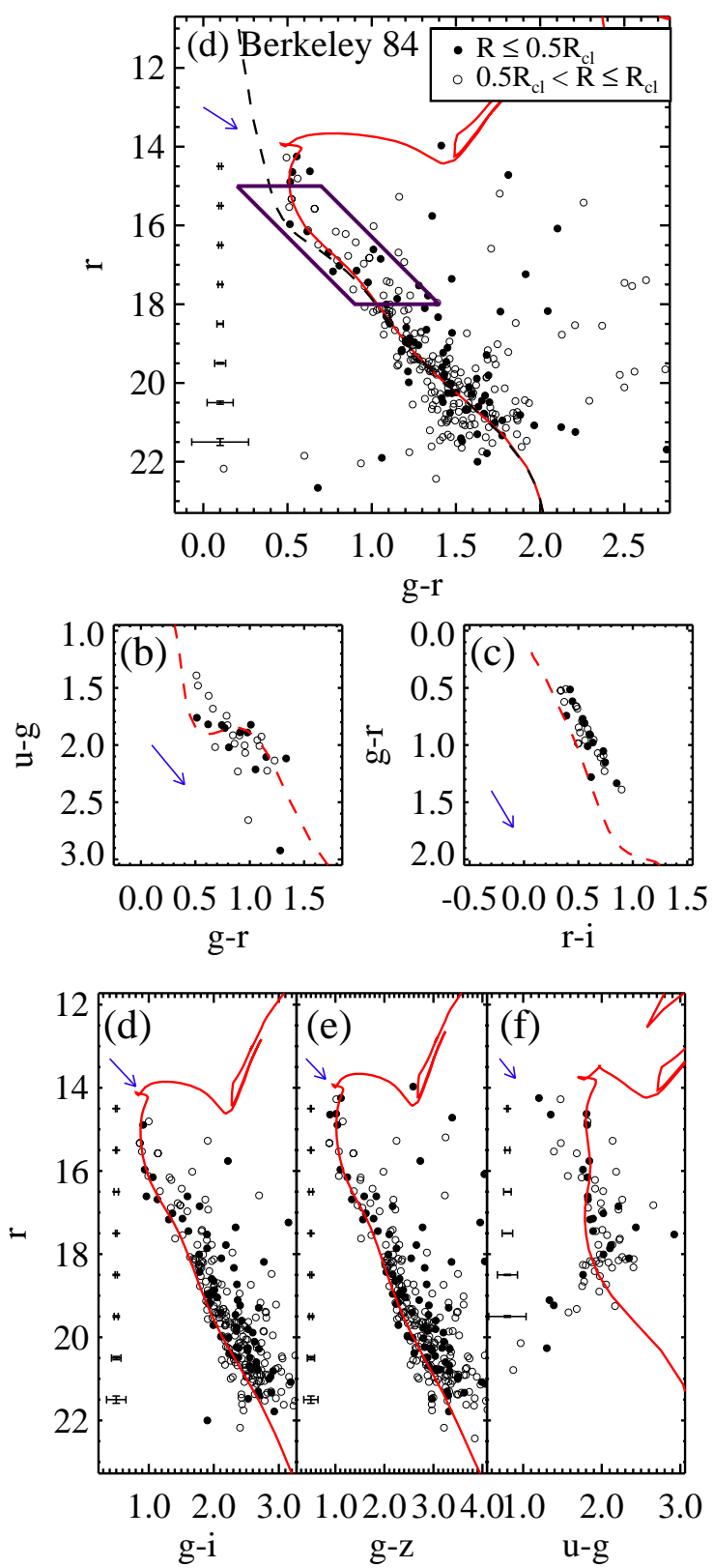

Fig. 12.- CMDs and CCDs of Berkeley 84. Symbols represent the same as in Fig. 11. The solid line represents an isochrone for $\log ($ age $[\mathrm{yr}])=8.65$ and $Z=0.019$, and the dashed line represents the ZAMS. The arrow in each panel represents a reddening vector. (a) The isochrone fitting for Berkeley 84. The isochrone is shifted according to $E(B-V)=0.73$ and $(m-M)_{0}=12.2$. The inclined solid box contains main sequence stars which are plotted in the following CCDs. (b) $(u-g)-(g-r)$ CCD. (c) $(g-r)-(r-i)$ CCD. (d) $r-(g-i)$ CMD. (e) $r-(g-z)$ CMD. (f) $r-(u-g)$ CMD.

\subsection{Pfleiderer 3}

The coordinates for the center of Pfleiderer 3 ( $\alpha=$ $23 \mathrm{~h} 08 \mathrm{~m} 11.8 \mathrm{~s}, \delta=+60^{\circ} 51^{\prime} 54^{\prime \prime}$ ) derived in this study are different by $12^{\prime \prime} .0$ in RA and $-30^{\prime \prime}$ in Dec from the values in DAML02. From the radial number density profile (Fig. 3e), we determined the radius of Pfleiderer 3 as $R_{\mathrm{cl}}=2^{\prime} .00 \pm 0.13$.

Pfleiderer 3 is not clearly visible in the gray-scale map of $r$ band image (Fig. 1e). However, the $r-(g-r)$ CMD of Pfleiderer 3 shows an obvious red giant clump at $(g-r) \simeq 2.2$ and $r \sim 19$ (Fig. 13a). Therefore, the blue and bright part of the main sequence around $(g-r) \simeq 1.0$ possibly consist of foreground stars, as shown in the CMD of the control field (Fig. 13b).

Fig. 14 shows the result of the isochrone fitting. We derived its age, $\log ($ age $[\mathrm{yr}])=9.0$, the reddening, $E(B-$ $V)=1.50 \pm 0.05$, and distance modulus, $(m-M)_{0}=$ $13.3 \pm 0.2$ from the $r-(g-r)$ CMD. Values derived from all other CMDs agree with those values. We also derived the reddening and distance modulus for the foreground sequence using the ZAMS. The reddening is $E(B-V)=1.40 \pm 0.05$ and the distance modulus is $(m-M)_{0}=12.95 \pm 0.2$. Fig. 15 displays the $(u-g)-$ $(g-r)$ CCD and $(g-r)-(r-i)$ CCD for the bright foreground sequence stars and the red giant clump stars located within two boxes in Fig. 14(a). The red giant clump stars are too faint at the $u$ band, so that they do not appear in the $(u-g)-(g-r)$ CCD (Fig. 15a) and neither does the $r-(u-g)$ CMD (Fig. 14d). Fig. 15b shows the $(g-r)-(r-i)$ CCD. There are two groups of stars: the blue one is the foreground sequence stars and the red one is the red giant clump stars.

\section{DISCUSSION}

\subsection{Comparison with Previous Studies}

The parameters of the clusters derived in this study are summarized in Table 8. Three clusters (Czernik 5, Berkeley 49 and Berkeley 84) among our targets were studied previously (Tadross 2008, 2009; Camargo et al. 2009; Subramaniam et al. 2010). We compare the parameters we obtained for these three clusters with those in the previous studies.

\section{Table 7.}

Estimates for reddenings and distance moduli of Berkeley 84 with different colors

\begin{tabular}{ccc}
\hline \hline $\mathrm{CMD}$ & $E(B-V)$ & $(m-M)_{0}$ \\
\hline$r-(g-r)$ & $0.73 \pm 0.06$ & $12.2 \pm 0.2$ \\
$r-(g-i)$ & $0.80 \pm 0.06$ & $12.2 \pm 0.2$ \\
$r-(g-z)$ & $0.73 \pm 0.06$ & $12.2 \pm 0.2$ \\
$r-(u-g)$ & $0.65 \pm 0.06$ & $12.2 \pm 0.2$ \\
\hline
\end{tabular}




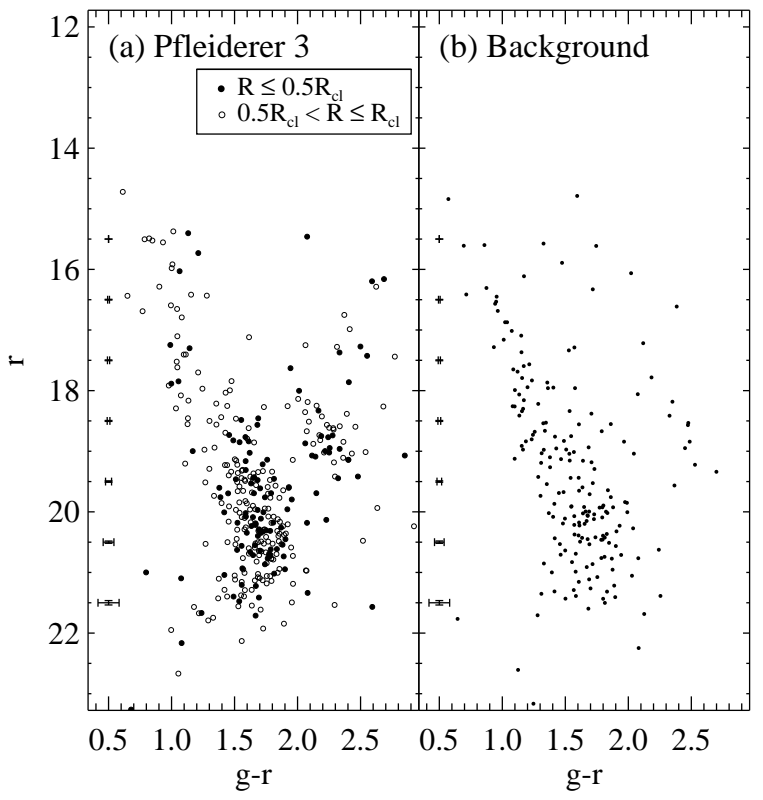

Fig. 13. - (a) $r-(g-r)$ CMD of the stars in Pfleiderer 3. Open circles are stars inside the radius of Pfleiderer 3 and filled dots are stars inside the half radius of the cluster. (b) $r-(g-r) \mathrm{CMD}$ of stars in the background region, which is shown in Fig. 1e. Mean photometric errors are marked on the left side of each panel.

In the case of Czernik 5, Tadross (2009) determined its age to be 700 Myrs (log $($ age $[y r])=8.85)$, considering the existence of some red giant branch stars in the CMD based on 2MASS photometry. This value is much larger than our estimate, $280 \pm 100 \mathrm{Myr}$. This large difference is probably due to the fact that we used the cluster coordinates derived in this study, which are different from those used by Tadross $(2009)-560^{\prime \prime}$ in RA and $81^{\prime \prime}$ in Dec.

The physical parameters of Berkeley 49 were derived by Tadross (2008) from 2MASS photometry, and by Subramaniam et al. (2010) from BVI photometry. We obtain the value for the age of Berkeley 49 to be $794 \pm 210$ Myrs $(\log ($ age $[y r])=8.9 \pm 0.13)$, while Tadross (2008) derived 160 Myrs $(\log ($ age $[\mathrm{yr}])=8.2)$ and Subramaniam et al. (2010) obtained $270 \pm 46$ Myrs $(\log ($ age $[y r])=8.43 \pm 0.07)$. Both of these previous studies estimated the age of Berkeley 49 to be younger than our result.

The age difference between ours and Tadross (2008) may be due to the fact that we included RGB stars as the members, while Tadross (2008) did not. These stars, shown in Fig. 16, have similar proper motions to those of other members of Berkeley 49 and so we have considered them to be the members of this cluster. It is noted that the four brightest RGB stars $(J \leq$ $11.2)$ are located within the half radius of Berkeley $4 \overline{9}$.
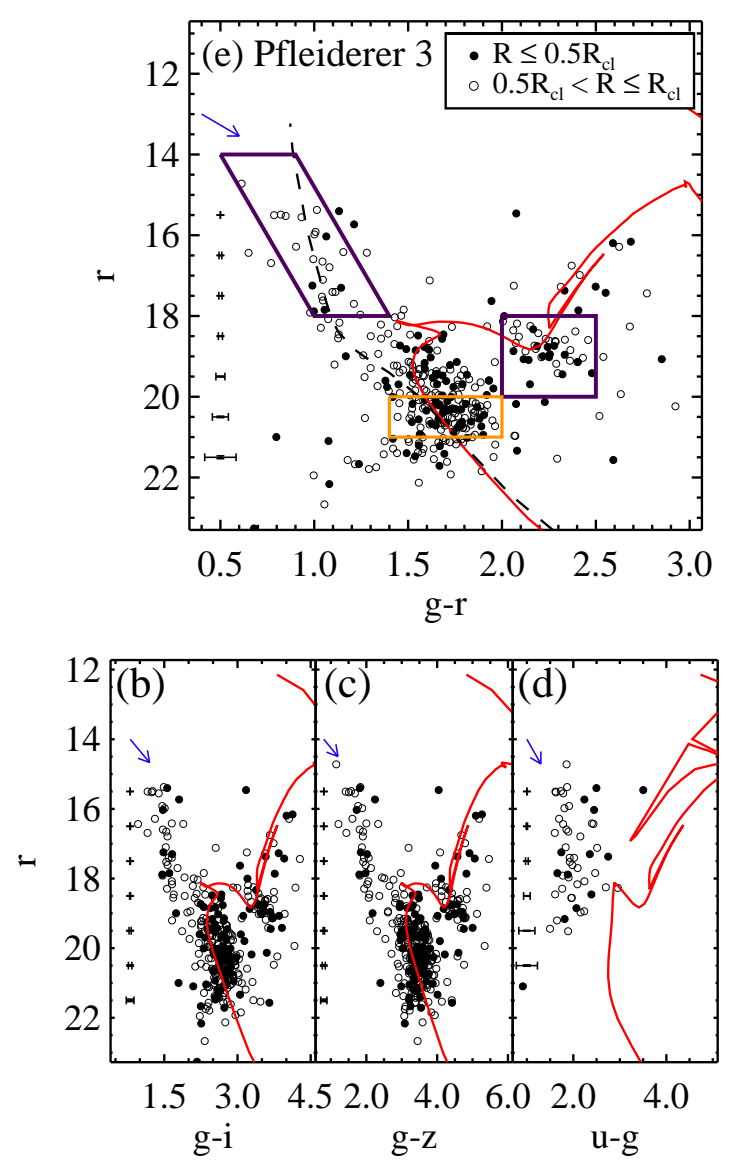

Fig. 14. - CMDs of Pfleiderer 3. Symbols represent the same as in Fig. 13. The arrow in each panel represents a reddening vector. (a) An isochrone fitting for Pfleiderer 3. Solid line represents an isochrone for $\log ($ age $[\mathrm{yr}])=9.0$ and $Z=0.016$. It is shifted according to $E(B-V)=1.5$ and $(m-M)_{0}=13.3$. Dashed line represents the ZAMS, that is shifted according to $E(B-V)=1.4$ and $(m-M)_{0}=12.95$. The inclined solid parallelogram at the left sequence contains foreground main sequence stars, and a solid square at the right contains red giant clump stars. Those stars are plotted in the following CCDs. Another square, located within $20<r<21$, contains main sequence stars. Radial distributions of stars located within each region are discussed in Section 5.4. (b) $r-(g-i)$ CMD. (c) $r-(g-z)$ CMD. (d) $r-(u-g)$ CMD.

Therefore, we consider them as members of the cluster, while Tadross (2008) regarded these stars as field stars.

The age difference between ours and Subramaniam et al. (2010) may be due to a difference in the turnoff point in the CMD. We considered the stars at $r \simeq$ $16.0 \mathrm{mag}$ and $(g-r) \simeq 1.1$ to be at the turnoff point, and considered nine brighter stars above this turnoff to be field stars. In contrast, Subramaniam et al. (2010) considered the turnoff point to be at $V \simeq 15 \mathrm{mag}$ and $(B-V) \simeq 1.2$, (corresponding to $r \simeq 14.6 \mathrm{mag}$ and $(g-r) \simeq 1.0$ (Jester et al. 2005)), which is $1.4 \mathrm{mag}$ 

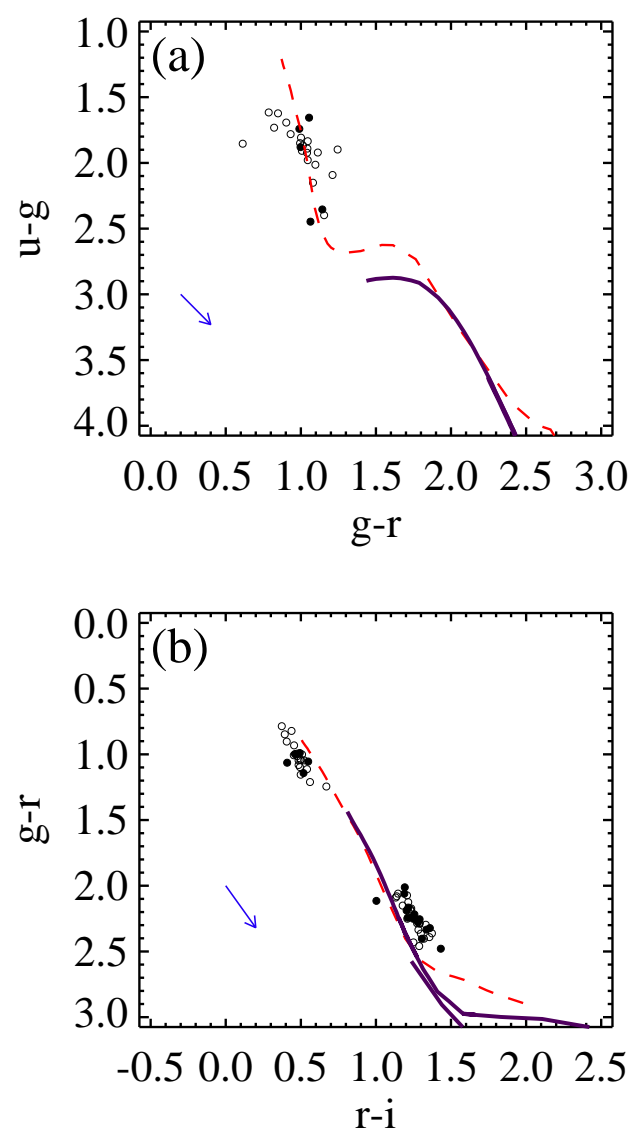

Fig. 15. - CCDs of Pfleiderer 3. Symbols represent the same as in Fig. 13. The arrow in each panel represents a reddening vector. (a) $(u-g)-(g-r)$ CCD of the stars in Pfleiderer 3. (b) $(g-r)-(r-i)$ CCD of stars in Pfleiderer 3. The dashed line in each CCD represents the ZAMS shifted according to $E(B-V)=1.40$ and the solid line represents the giant part of an isochrone for $\log ($ age $[\mathrm{yr}])=9.0$ shifted according to $E(B-V)=1.50$.

brighter than the turnoff we chose.

The difference in age estimates may depend on the reddening difference. Our reddening estimate, $E(B-V)=1.18$, is slightly smaller than the values given by Tadross (2008) and Subramaniam et al. (2010), $E(B-V)=1.57$ and $E(B-V)=1.35$, respectively. Therefore, the larger values for ages are obtained when we choose smaller values for reddenings.

In spite of these reddening and age differences, estimated distances are consistent with each other within the error. Our distance is $2090 \pm 200 \mathrm{pc}\left((m-M)_{0}=\right.$ $11.6 \pm 0.2)$, while the distance of Tadross (2008) is $2035 \pm 110 \mathrm{pc}\left((m-M)_{0}=11.54 \pm 0.12\right)$ and that of Subramaniam et al. (2010) is $2300 \pm 230 \mathrm{pc}((m-M))_{0}=$ $11.81 \pm 0.22)$.

We also compared the physical parameters of Berkeley 84 with those in Tadross (2008) and Camargo et al.
(2009), using the 2MASS CMDs which are shown in Fig. 17. Both of the previous studies regarded four RGB stars around $J \simeq 11.5 \mathrm{mag}$ and $(J-H) \simeq 0.8$ as field stars, and considered that two blue stars around $J \simeq 11.5 \mathrm{mag}$ and $(J-H) \simeq 0.2$ to be the members. However, the brightest RGB star with $J \simeq 9 \mathrm{mag}$ and two RGB stars with $J \simeq 11.5$ mag are located within the half radius of Berkeley 84, while the two blue stars are located at the distance farther than the half radius. Therefore, the RGB stars are considered to have a higher probability of being the members than the blue stars. This different member selection is a reason for the different estimates of reddenings. Our reddening value for $E(B-V)=0.73 \pm 0.06$ is consistent with the value of Tadross (2008), $E(B-V)=0.76 \pm 0.1$. Our estimate is much larger than the value derived by Camargo et al. (2009), $E(B-V)=0.58 \pm 0.06$.

The reason for age differences of Berkeley 84 seems to be similar to the case for Berkeley 49. We derived the age of Berkeley $84,447 \pm 130$ Myrs $(\log (\operatorname{age}[y r])=8.65 \pm$ 0.15). Tadross (2008) and Camargo et al. (2009) estimated the age to be 120 Myrs $(\log (\operatorname{age}[y r])=8.1)$ and $360 \pm 50$ Myrs $(\log (\operatorname{age}[\mathrm{yr}])=8.55 \pm 0.06)$, respectively Tadross (2008)'s value is much smaller than ours, while Camargo et al. (2009)'s value is consistent with ours, within the error. Our distance estimate, $2750 \pm 270$ pc $\left((m-M)_{0}=12.2 \pm 0.2\right)$, is larger than $2025 \pm 95$ pc $\left((m-M)_{0}=11.55 \pm 0.12\right)$ of Tadross (2008) and $1700 \pm 100 \mathrm{pc}\left((m-M)_{0}=11.15 \pm 0.13\right)$ of Camargo et al. (2009).

\subsection{Spatial Distribution of the Open Clusters}

With the distance estimates for our target clusters we can investigate where they are located in the Galactic plane. In Fig. 18a we show the spatial distribution of these clusters in the galactic plane. We also plotted the positions of intermediate-age clusters in DAML02 for comparison. The age distribution of the clusters in DAML02 shows three groups: young clusters with $\log (\operatorname{age}[\mathrm{yr}])<7.4$, intermediateage clusters with $\log ($ age $[\mathrm{yr}])=7.4-9.0$, and old clusters with $\log (\operatorname{age}[\mathrm{yr}])>9.0$. The age range for the intermediate-age clusters covers the ages of our target clusters. Therefore, we plotted the intermediateage clusters with $\log ($ age $[\mathrm{yr}])=7.4-9.0$. In addition we marked the spiral arms in our Galaxy (Churchwell et al. 2009). The intermediate-age clusters are mostly located near the Sun, and they do not show any significant correlation with the arms. Berkeley 49 and Berkeley 84 are located in the Orion spur, Czernik 5 is in the Perseus arm, and Pfleiderer 3 and Alessi 53 are located between the Perseus arm and the outer arm.

Fig. 18b displays the distance from the Galactic plane $(z)$ versus the galactocentric distance $\left(R_{\mathrm{GC}}\right)$ for the target clusters. We also plotted the same for the intermediate-age clusters in DAML02 for comparison. All the target clusters are closer than $94 \mathrm{pc}$ to the Galactic plane. 
Table 8.

Physical parameters of the clusters

\begin{tabular}{|c|c|c|c|c|c|c|}
\hline Name & $\begin{array}{l}\text { Age } \\
\text { Myr }\end{array}$ & $\begin{array}{c}E(B-V) \\
\quad \operatorname{mag}\end{array}$ & $\begin{array}{c}\text { Distance } \\
\text { pc }\end{array}$ & $\begin{array}{l}R_{\mathrm{GC}}^{\mathrm{a}} \\
\mathrm{kpc}\end{array}$ & $\begin{array}{l}\mathrm{z}^{\mathrm{b}} \\
\mathrm{pc}\end{array}$ & Reference \\
\hline Czernik 5 & $\begin{array}{c}280 \pm 100 \\
700\end{array}$ & $\begin{array}{c}1.20 \pm 0.05 \\
1.23\end{array}$ & $\begin{array}{l}2750 \pm 270 \\
2205 \pm 100\end{array}$ & $\begin{array}{l}10.01 \pm 0.20 \\
9.57 \pm 0.07\end{array}$ & $\begin{array}{l}-27 \pm 3 \\
-23^{\mathrm{c}}\end{array}$ & $\begin{array}{c}\text { This study } \\
\text { Tadross (2009) }\end{array}$ \\
\hline Alessi 53 & $250 \pm 150$ & $0.80 \pm 0.05$ & $4570 \pm 330$ & $12.35 \pm 0.67$ & $-53 \pm 4$ & This study \\
\hline Berkeley 49 & $794 \pm 210$ & $1.18 \pm 0.05$ & $2090 \pm 200$ & $7.58 \pm 0.01$ & $94 \pm 9$ & This study \\
\hline & $\begin{array}{c}160 \\
270 \pm 46\end{array}$ & $\begin{array}{c}1.57 \pm 0.1 \\
1.35 \pm 0.02\end{array}$ & $\begin{array}{l}2035 \pm 110 \\
2300 \pm 230\end{array}$ & $\begin{array}{l}7.58 \pm 0.01 \\
7.57 \pm 0.01\end{array}$ & $\begin{array}{c}91^{\mathrm{c}} \\
19 \pm 2\end{array}$ & $\begin{array}{l}\text { Tadross (2008) } \\
\text { Subramaniam et al. (2010) }\end{array}$ \\
\hline Berkeley 84 & $\begin{array}{c}447 \pm 130 \\
120\end{array}$ & $\begin{array}{c}0.73 \pm 0.06 \\
0.76 \pm 0.1\end{array}$ & $\begin{array}{c}2750 \pm 270 \\
2025 \pm 95\end{array}$ & $\begin{array}{l}7.57 \pm 0.01 \\
7.58 \pm 0.01\end{array}$ & $\begin{array}{c}61 \pm 6 \\
45^{\mathrm{c}}\end{array}$ & $\begin{array}{c}\text { This study } \\
\text { Tadross }(2008)\end{array}$ \\
\hline & $360 \pm 50$ & $0.58 \pm 0.06$ & $1700 \pm 100$ & $7.61 \pm 0.01$ & $38 \pm 3$ & Camargo et al. (2009) \\
\hline Pfleiderer 3 & $1000 \pm 260$ & $1.50 \pm 0.05$ & $4570 \pm 440$ & $10.53 \pm 0.31$ & $37 \pm 4$ & This study \\
\hline
\end{tabular}

all $R_{\mathrm{GC}}$ are calculated using $R_{\odot}=8 \mathrm{kpc}$.

${ }^{\mathrm{b}}$ These values represent heights from the galactic plane. All these values have been calculated using their distances, except Tadross (2008) and Tadross (2009).

${ }^{\mathrm{c}}$ These values are presented in the references.
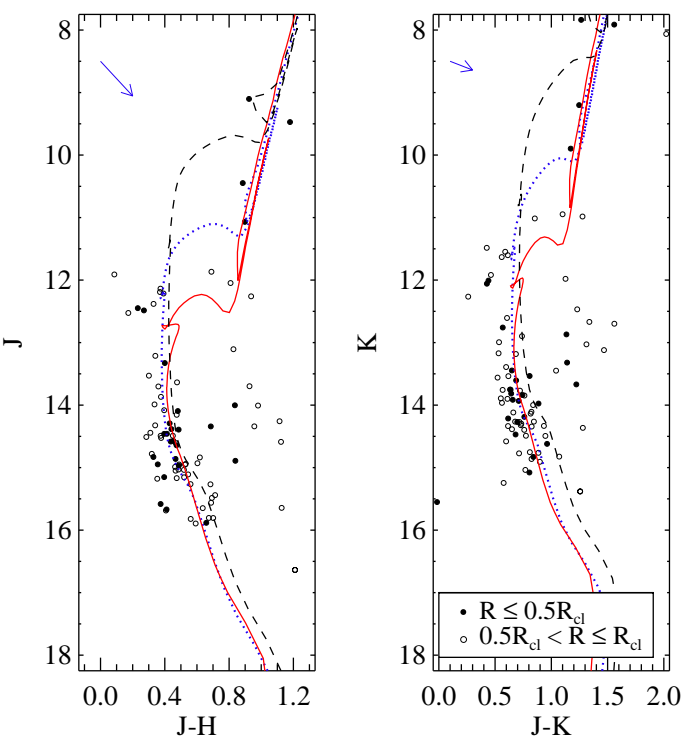

Fig. 16. - Left. $J-(J-H)$ CMD of the stars in Berkeley 49. Right. $K-(J-K)$ CMD. Solid line represents the isochrone for the same parameters as determined in the $r-(g-r)$ CMD (Fig. 10a). Dashed line and dotted line represent the isochrones for the parameters given in Tadross (2008) and Subramaniam et al. (2010), respectively.
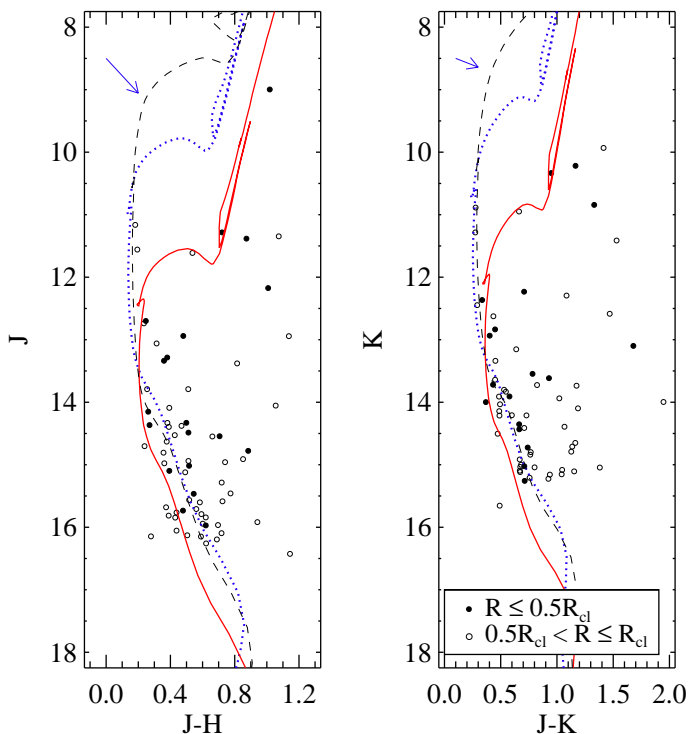

Fig. 17. - The same as Fig. 16 but for Bekeley 84. Dashed line and dotted line represent the isochrones for the parameters given in Tadross (2008) and Camargo et al. (2009), respectively. 

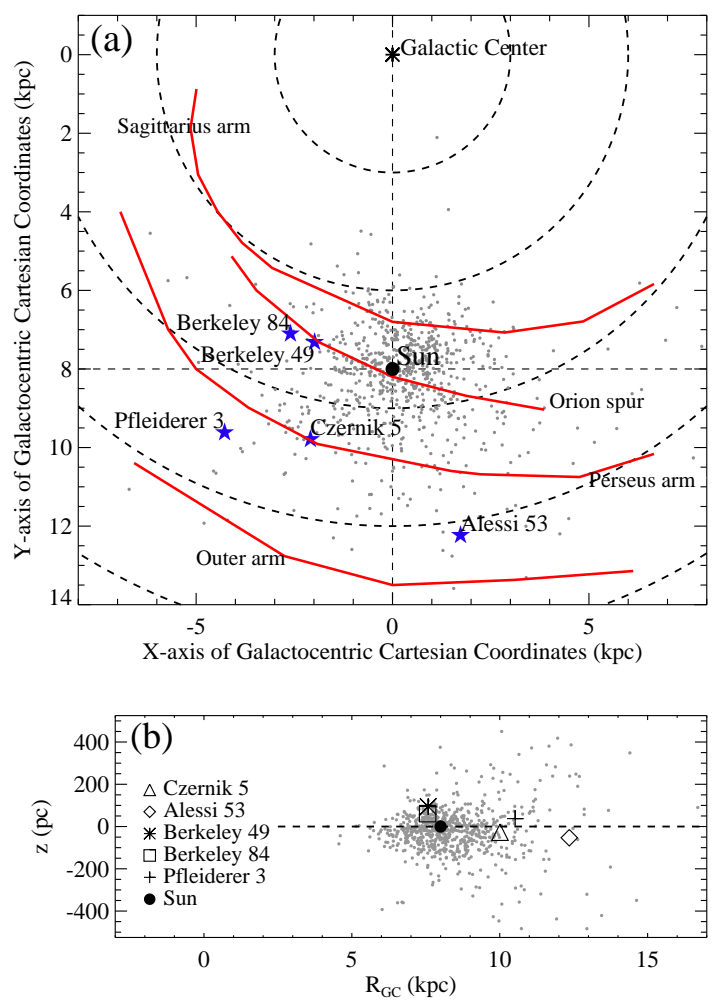

Fig. 18. - (a) The spatial distribution of the clusters (star symbols) in the Galactic plane. Dots represent the intermediate-age clusters $(7.4<\log ($ age $[\mathrm{yr}])<9.0)$ in DAML02. The Sun is marked by a filled circle at the center. The radii of the dashed line circles are $3,6,9,12$, and $15 \mathrm{kpc}$. Curved solid lines represent spiral arms. (b) The vertical distribution of the clusters with respect to the Galactic plane (dashed line). Dots represent the same as in (a). Large symbols represent the target clusters and the Sun, respectively.

\subsection{The Blue Stars in Berkeley 49}

We found nine blue stars in Berkeley 49. These stars are bluer than the turnoff point of Berkeley 49 and redder than the ZAMS. Subramaniam et al. (2010) considered these stars to be the most bright main sequence stars of Berkeley 49. However, they seem to be neither the main sequence stars nor blue straggler stars, as described in the following.

It is generally known that blue straggler stars are heavier than main sequence stars. Therefore, if these blue stars are blue straggler stars or main sequence stars, then it is expected that they show a stronger or similar central concentration than the other main sequence stars. We investigated this using two ways: the spatial distribution of the stars, and the cumulative distribution functions (CDFs) for radial distributions of the stars. We selected three groups of stars in this cluster for the analysis: the blue stars, the bright main sequence stars, and the faint main sequence stars, according to the marked regions in Fig. 10a.

Fig. 19 shows the spatial distribution of these three groups of stars marked in a gray-scale map of Berkeley 49. The bright main sequence stars show a stronger central concentration than the other two groups. It is also seen that both the blue stars and the faint main sequence stars are distributed sparsely all over the cluster region.

In Fig. 20 we plotted the CDFs for radial distributions of the three groups of stars. It is seen that the bright main sequence stars are more centrally concentrated than the other two groups, and that these two groups show similar distributions. We performed Kolmogorov-Smirnov (K-S) tests to estimate how much these groups are different in radial distribution. The tests yield $19 \%, 72 \%$, and $7 \%$ probabilities for the sets of (blue stars - bright main sequence stars), (blue stars - faint main sequence stars) and (bright - faint main sequence stars), respectively. These probabilities indicate that the two main sequence groups are clearly different. However it is not obvious that the blue stars are different from the other two groups. This is not consistent with the dynamic prediction for the blue straggler stars. Therefore, the blue stars must be considered to be field stars.

\subsection{The Blue Sequence of Pfleiderer 3}

We found a blue sequence in the CMD of Pfleiderer 3 (Fig. 14a). We selected the blue sequence stars, the red giant clump stars and the main sequence stars in this cluster, to check the membership of the blue sequence stars according to the marked regions in Fig. 14a.

Fig. 21 shows CDFs for radial distributions of the blue sequence stars, the red giant clump stars and the main sequence stars in Pfleiderer 3. The red giant clump stars and the main sequence stars show a stronger central concentration than the blue sequence stars. Therefore, these blue sequence stars are considered to be foreground stars. K-S tests yield $27 \%$ probability for the set of (blue sequence stars - red giant clump stars) and $66 \%$ probability for the set of (blue sequence stars - main sequence stars). These probabilities indicate that the blue sequence stars are a different population from the red giant clump stars and the main sequence stars.

According to the estimates of the distance and foreground reddening of this blue sequence, the blue sequence stars, being 680 pc closer to the Sun than Pfleiderer 3, are located in the Perseus arm, while Pfleiderer 3 is between the Perseus arm and the outer arm. It is consistent with the location of Pfleiderer 3 which is located beyond the Perseus arm. 


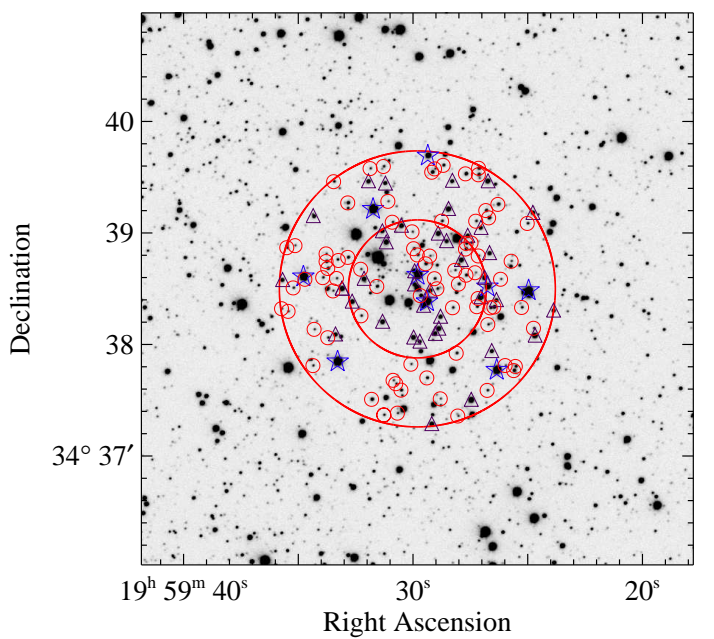

Fig. 19. - A gray-scale map of Berkeley 49. The large solid circle represents the radius of the cluster and the small circle represents the half radius. Stars, triangles and open circles represent the blue stars, bright main sequence stars and faint main sequence stars, respectively.

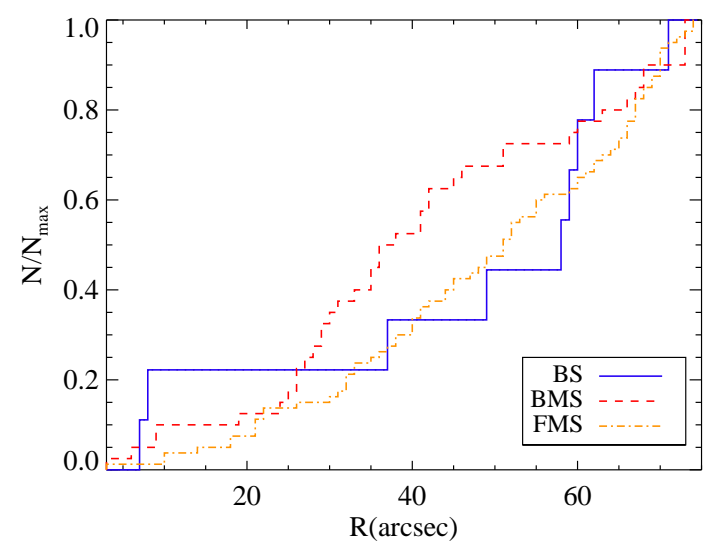

Fig. 20.- Cumulative number distributions of stars in Berkeley 49. The solid, dashed and dash-dot line represent the blue stars, bright main sequence stars and faint main sequence stars, respectively. The numbers of the stars are normalized by the maximum value.

\section{SUMMARY}

We have derived the physical parameters of five open clusters in the SDSS. To obtain parameters, we determine the position of these clusters first. Then we exclude fast proper motion stars using the velocity dispersion of each cluster using the PPMXL. The size of the clusters is determined using the remaining stars. We assume the metallicity of these clusters to follow

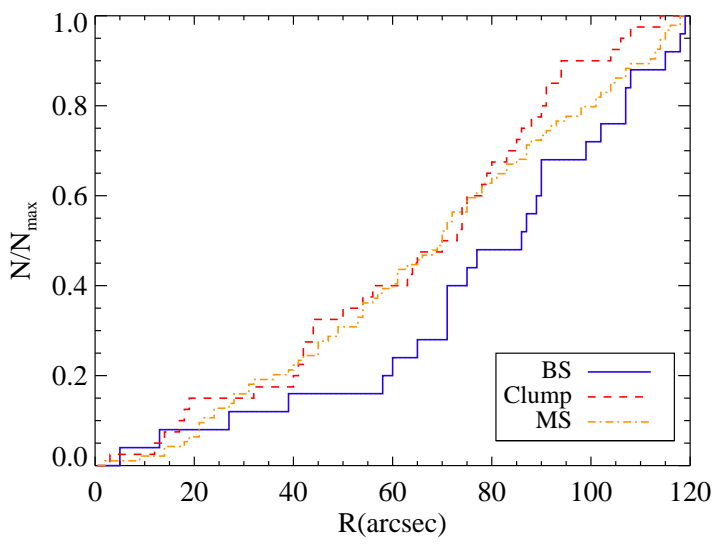

Fig. 21. - Cumulative number distributions of stars in Pfleiderer 3. The solid and dashed line represent the blue sequence stars and the red giant clump stars, respectively. The numbers of the stars are normalized by the maximum value.

the radial metallicity gradient of OCs using data in DAML02. We determine the age, distance and reddening of the clusters using the Padova isochrones. The ages of the clusters are in the range from 250 to 1000 Myrs, the distance to these clusters are derived to be $2.0-4.4 \mathrm{kpc}$. The reddenings are estimated to be $E(B-V)=0.71-1.55$. The derived physical parameters are listed in Table 8.

These clusters follow the distribution of the intermediate-age OCs without exception. They are located from the Orion spur to between the Perseus arm and the outer arm. The distance from the galactic plane for the clusters is less than $94 \mathrm{pc}$.

We find nine stars which are bluer than the turnoff point of Berkeley 49. We compare their central concentration with that of bright and faint main sequence stars. The blue stars are not more centrally concentrated than the bright main sequence stars, but are distributed sparsely all over the cluster region. Therefore, these blue stars are considered to be field stars.

There are many blue sequence stars in Pfleiderer 3 , but they seem to be foreground stars. Physical parameters of these blue sequence stars are derived from the ZAMS fitting. The reddening is derived to be $E(B-V)=1.40 \pm 0.05$ and distance modulus is $(m-M)_{0}=12.95 \pm 0.2$. This is corresponding to the distance to the Perseus arm lying in the foreground of the cluster.

\section{ACKNOWLEDGMENTS}

This work was supported by Mid-career Researcher Program through NRF grant funded by the MEST (No.2010-0013875). This research was also supported by the BK21 program of the Korean Government. 


\section{REFERENCES}

Abazajian, K. N., et al. 2009, The Seventh Data Release of the Sloan Digital Sky Survey, ApJS, 182, 543

Ahumada, J. A., \& Lapasset, E. 2007, New Catalogue of Blue Stragglers in Open Clusters, A\&A, 463, 789

An, D., et al. 2008, Galactic Globular and Open Clusters in the Sloan Digital Sky Survey. I. CrowdedField Photometry and Cluster Fiducial Sequences in Ugriz, ApJS, 179, 326

An, D., et al. 2009, Galactic Globular and Open Clusters in the Sloan Digital Sky Survey. II. Test of Theoretical Stellar Isochrones, ApJ, 700, 523

Andreuzzi, G., Bragaglia, A., Tosi, M., \& Marconi, G. 2010, Old Open Clusters and the Galactic Metallicity Gradient: Berkeley 20, Berkeley 66, and Tombaugh 2, MNRAS, 412, 1265

Ann, H. B., et al. 2002, BOAO Photometric Survey of Galactic Open Clusters. II. Physical Parameters of 12 Open Clusters, AJ, 123, 905

Bertin, E., Mellier, Y., Radovich, M., Missonnier, G., Didelon, P., \& Morin, B. 2002, The TERAPIX Pipeline, ASPC, 281, 228

Camargo, D., Bonatto, C., \& Bica, E. 2009, Astrophysical Parameters of 14 Open Clusters Projected Close to the Galactic Plane, A\&A, 508, 211

Carraro, G., Vázquez, R. A., \& Moitinho, A. 2008, Blue Straggler Stars in Galactic Open Clusters and the Effect of Field Star Contamination, A\&A, 482, 777

Chen, L., Hou, J. L., \& Wang, J. J. 2003, On the Galactic Disk Metallicity Distribution from Open Clusters. I. New Catalogs and Abundance Gradient, AJ, 125,1397

Churchwell, E. et al. 2009, The Spitzer/GLIMPSE Surveys: A New View of the Milky Way, PASP, 121, 213

Dias, W. S., Alessi, B. S., Moitinho, A., \& Lépine, J. R. D. 2002, New Catalogue of Optically Visible Open Clusters and Candidates, A\&A, 389, 871

Friel, E. D., et al. 2002, Metallicities of Old Open Clusters, AJ, 124, 2693

Froebrich, D., Scholz, A., \& Raftery, C. L. 2007, A Systematic Survey for Infrared Star Clusters with $|b|<20$ Using 2MASS, MNRAS, 374, 399

Girardi, L., Grebel, E. K., Odenkirchen, M., \& Chiosi, C. 2004, Theoretical Isochrones in Several Photometric Systems II. The Sloan Digital Sky Survey Ugriz System, A\&A, 422, 205

Jester, S. et al. 2005, The Sloan Digital Sky Survey View of the Palomar-Green Bright Quasar Survey, AJ, 130,873
Kronberger, M., et al. 2006, New Galactic Open Cluster Candidates from DSS and 2MASS Imagery, A\&A, 447, 921

Koposov, S. E., Glushkova, E. V., \& Zolotukhin, I. Y. 2008, Automated Search for Galactic Star Clusters in Large Multiband Surveys. I. Discovery of 15 New Open Clusters in the Galactic Anticenter Region, A\&A, 486, 771

Lee, M. G. 1997, UBVI CCD Photometry of the Remote Old Open Cluster AM-2, AJ, 113, 2

Lupton, R. H., Ivezic, Z., Gunn, J. E., Knapp, G., Strauss, M. A., \& Yasuda, N. 2002, SDSS Imaging Pipelines, SPIE, 4836, 350

Mapelli, M., Sigurdsson, S., Ferraro, F. R., Colpi, M., Possenti, A., \& Lanzoni, B. 2006, The Radial Distribution of Blue Straggler Stars and the Nature of Their Progenitors, MNRAS, 373, 361

Park, H. S., \& Lee, M. G. 1999, UBVI Charge-Coupled Device Photometry of Two Old Open Clusters NGC 1798 and 2192, MNRAS, 304, 883

Piatti, A. E., 2010, Evidence of Enhanced Formation Episodes in the Galactic Open Cluster System, A\&A, 513, 13

Roeser, S., Kharchenko, N. V., Piskunov, A. E., Schilbach, E., Scholz, R. -D., \& Zinnecker, H. 2010, Open Clusters and the Galactic Disk, AN, 331, 519

Roeser, S., Demleitner, M., \& Schilbach, E. 2010, The PPMXL Catalog of Positions and Proper Motions on the ICRS. Combining USNO-B1.0 and 2MASS, AJ, 139, 2440

Skrutskie, M. F., et al. 2006, The Two Micron All Sky Survey (2MASS), AJ, 131, 1163

Stetson, P. B. 1987, DAOPHOT - A Computer Program for Crowded-Field Stellar Photometry, PASP, 99, 191

Subramaniam, A., Carraro, G., \& Janes, K. A. 2010, Optical Photometry and Basic Parameters of 10 Unstudied Open Clusters, MNRAS, 404, 1385

Tadross, A. L. 2008, A Catalogue of Previously Unstudied Berkeley Clusters, MNRAS, 389, 285

Tadross, A. L. 2009, The Fundamental Parameters of 60 Unstudied Open Star Clusters (Czernik, DolDzim, Kronberger, and Turner), Ap\&SS, 323, 383

York, D. G., et al. 2000, The Sloan Digital Sky Survey: Technical Summary, AJ, 120, 1579 